\title{
The Baryon Content of Extremely Low Mass Dwarf Galaxies
}

\author{
M. Geha ${ }^{1}$ \\ The Observatories of the Carnegie Institute of Washington, 813 Santa Barbara St., \\ Pasadena,CA 91101.mgeha@ociw.edu \\ M. R. Blanton, M. Masjedi \\ Center for Cosmology and Particle Physics, New York University, New York, NY 10003. \\ blanton@nyu.edu,morad.masjedi@physics.nyu.edu \\ A. A. West \\ Astronomy Department, University of California, 601 Campbell Hall, Berkeley, CA 94720. \\ awest@astron.berkeley.edu
}

\begin{abstract}
We investigate the gas content and baryonic Tully-Fisher relationship for extremely low luminosity dwarf galaxies in the absolute magnitude range $-13.5>$ $M_{r}-5 \log _{10} h_{70}>-16$. The sample is selected from the Sloan Digital Sky Survey and consists of 101 galaxies for which we have obtained follow-up HI observations using the Arecibo Observatory and Green Bank Telescope. This represents the largest homogeneous sample of dwarf galaxies at low luminosities with well-measured HI and optical properties. The sample spans a range of environments, from dense groups to truly isolated galaxies. The average neutral gas fraction is $\left\langle f_{\text {gas }}\right\rangle=0.6$, significantly exceeding that of typical gas-rich galaxies at higher luminosities. Dwarf galaxies are therefore less efficient at turning gas into stars over their lifetimes. The strong environmental dependence of the gas fraction distribution demonstrates that while internal processes can reduce the gas fractions to roughly $f_{\text {gas }}=0.4$, external processes are required to fully remove gas from a dwarf galaxy. The average rotational velocity of our sample is $\left\langle v_{\text {rot }}\right\rangle=$ $\left\langle\mathrm{W} 20_{i, t} / 2\right\rangle=50 \mathrm{~km} \mathrm{~s}^{-1}$ based on HI line-widths. In this luminosity range, the optical Tully-Fisher relationship has significantly more scatter compared to the baryonic relationship. Including more massive galaxies from the literature, we fit a baryonic Tully-Fisher slope of $M_{\text {baryon }} \propto v_{\text {rot }}^{3.70 \pm 0.15}$. This slope compares well
\end{abstract}

\footnotetext{
${ }^{1}$ Hubble Fellow.
} 
with CDM models that assume an equal baryon to dark matter ratio at all masses. While gas stripping or other processes may modify the baryon to dark matter ratio for dwarfs in the densest environments, the majority of dwarf galaxies in our sample have not preferentially lost significant baryonic mass relative to more massive galaxies.

Subject headings: galaxies: dwarf — galaxies: kinematics and dynamics

\section{Introduction}

The properties of low mass dwarf galaxies, and their differences from luminous galaxies, provide important clues to understanding both cosmology and galaxy formation. As probes of galaxy formation, dwarf galaxies should be extremely sensitive to processes such as star formation feedback, ram pressure stripping, and tidal stripping. These processes are often invoked to explain properties of relatively luminous galaxies and should even more dramatically affect dwarf galaxies, which have smaller masses and shallower potential wells. As probes of cosmology, the number of dwarf galaxies has provided a challenge for cosmological models.

The current Cold Dark Matter model with a cosmological constant ( $\Lambda$ CDM; Spergel et al. 2006) makes robust predictions for the number of dark matter halos as a function

of mass (Sheth et al. 2001). However, extending this model to predict the number density of observed galaxies requires including physical effects such as gas cooling, star-formation, supernova feedback, etc., that are too complex to follow exactly in numerical predictions. It is clear that the number of galaxies as a function of luminosity is much shallower than the prediction for the number as a function of halo mass (Benson et al. 2003). The most extreme example of this problem is the "substructure problem" in the Local Group (Klypin et al. 1999; Moore et al. 1999). If we assume galaxies are associated one-to-one with dark matter halos and that $\Lambda \mathrm{CDM}$ is correct, the mass-to-light ratios of galaxies therefore must decline as a function of host halo mass. That is, the efficiency of forming stars and/or retaining gas must be much smaller for low mass galaxies.

A number of physical models have been proposed to explain high mass-to-light ratios in low mass galaxies, including photoevaporation of gas during reionization (Shapiro et al. 2004; Mayer \& Moore 2004), suppression of gas accretion during reionization (Gnedin 2000; Bullock et al. 2000; Benson et al. 2003), feedback from supernovae (Dekel \& Woo 2003; Nagashima \& Yoshii 2004), and ram pressure stripping of gas (Mori \& Burkert 2000). Some of these processes only occur for dwarf galaxies near a luminous galaxy and not for isolated 
ones (Kravtsov et al. 2004). The relative importance of these processes in the evolution of dwarf galaxies has significant implications for galaxies of all sizes at both low and high redshift. For example, the importance of galactic winds in low mass galaxies will strongly affect our understanding of the mass-metallicity relation (Tremonti et al. 2004; Lee et al. 2006) and the enrichment of the intergalactic medium at high redshift (Scannapieco et al. 2005). By comparing the gas content and Tully-Fisher relationships of dwarfs as a function of environment, it should be possible to determine the importance of "external" environmental effects like stripping relative to "internal" effects such as feedback.

Historically, extremely low luminosity nearby galaxies have been difficult to find because their colors and apparent sizes are similar to those of more luminous, and far more numerous, background galaxies. By far the best known set of dwarfs is that in the Local Group (Mateo 1998; Grebel et al. 2003; Dolphin et al. 2005), where the even the lowest mass dwarfs can be detected as over-densities in stellar counts (Willman et al. 2005; Zucker et al. 2004). The dwarf galaxy population has also been well studied in nearby clusters, such as Virgo and Fornax, where the number of dwarfs is large relative the background population (Binggeli et al. 1985; Ferguson \& Sandage 1991) or in other environments where dwarfs are expected, eg. as satellites to bright galaxies (Zaritsky et al. 1997; Gutiérrez et al. 2002). Assembling samples of dwarfs in more distant and less dense environments has been challenging. Using criteria such as surface brightness and irregularity, several authors have assembled large catalogs of nearby dwarf galaxies (Impey et al. 1996; Schombert et al. 2001; Hunter \& Elmegreen 2004; Karachentsev et al. 2004).

Addressing the above issues requires a large sample of galaxies across a range of environments, with minimal selection effects. Deep, wide-field optical spectroscopic surveys, such as the Sloan Digital Sky Survey (SDSS), provide a large, homogeneous sample of dwarf galaxies covering a wide range of galactic properties and environments. Concurrently, blind HI surveys such as the HI Parkes All Sky Survey (HIPASS; Meyer et al. 2004) and the upcoming Arecibo Legacy Fast ALFA Survey (ALFALFA; Giovanelli et al. 2005) will provide similarly large samples, with a very complementary set of selection effects.

Unlike present-day massive disk galaxies, which have used up much of their gas reservoirs (Read \& Trentham 2005), the gaseous component of dwarf galaxies cannot be ignored (Schombert et al. 2001; van Zee 2001). Indeed, we show that at the lowest luminosities dwarf galaxies are mostly neutral gas. A galaxy's gas content reflects the efficiency of star formation modified by processes which add or removal gas from the system such as gas infall or ram pressure stripping. The goal of this paper is to combine the optical properties, dynamics and neutral gas content of low mass dwarf galaxies in order to better understand the processes important in dwarf galaxy evolution. 
We present neutral hydrogen (HI) observations for 101 low luminosity dwarf galaxies selected with uniform and well-understood criteria from the SDSS. We describe the catalog from which these dwarfs were selected in $\S 2.1$, estimate the environment of each object in $\S 2.2$ and discuss the HI observations in $\S 2.3$. We investigate correlations between the gas fraction, color, star formation rate and environment of this sample in $\S 3.1$ and the TullyFisher relationship in $\S 3.2$. When necessary, we assume a Hubble constant of $\mathrm{H}_{0}=70 h_{70} \mathrm{Mpc}$ $\mathrm{km} \mathrm{s}^{-1}$ where $h_{70}=1$.

\section{Data}

\subsection{The Sample}

We selected dwarf galaxies with magnitude $M_{r}-5 \log _{10} h_{70}>-16$ for HI observations from the low luminosity galaxy catalog of Blanton et al. (2005), a subsample of the New York University Value-Added Galaxy Catalog (NYU-VAGC; Blanton et al. 2005b) based on the SDSS (York et al. 2000) Data Release 2 (DR2; Abazajian et al. 2004). The Blanton et al. (2005) catalog represents a significant improvement over simply selecting galaxies from the SDSS catalog, which is not optimized for nearby, low surface brightness galaxies. It is a flux-limited sample selected with an optical apparent magnitude limit of $m_{r} \sim 17.8$. For each galaxy, the catalog provides the SDSS redshift, multi-band photometry, structural measurements (in particular, two-dimensional Sérsic fits as described in Blanton et al.) and emission line measurements (Tremonti et al. 2004). Distances are estimated based on a model of the local velocity field (Willick et al. 1997). Distance errors have been folded into error estimations of all distance-dependent quantities such as absolute magnitude and HI mass.

There are two selection effects of the Blanton et al. (2005) catalog relevant to this analysis: (1) The SDSS flux limit at $m_{r} \sim 17.8$ restricts the detection of galaxies with $M_{r}-5 \log _{10} h_{70}=-16$ to within $60 \mathrm{Mpc}$ in distance $(20 \mathrm{Mpc}$ for the least luminous galaxies, $\left.M_{r}-5 \log _{10} h_{70}=-13.5\right)$. The catalog therefore does not span the full range of galaxy environments. While there are plenty of voids and groups in this volume, there are few very dense regions. For example, given the angular limits of the SDSS DR2, we see only the fringes of the Virgo Cluster. (2) The catalog does not include low surface brightness galaxies. As shown in Blanton et al. (2005), the SDSS completeness as a function of half-light surface brightness drops below $50 \%$ at $\mu_{50, r} \sim 23.5 \mathrm{mag} \operatorname{arcsec}^{-2}$. In the absolute magnitude range of interest, we would miss half of the dwarf galaxies in the Local Group (Mateo 1998) given this surface brightness cutoff. According to the definition of McGaugh (1996), 10\% of our sample galaxies are considered Low Surface Brightness (LSB) with $\mu_{o}^{B} \geq 22.7$. With the exception of 
these two selection effects, the Blanton et al. spectroscopic catalog is not selected by galaxy property in any particular way. For example, galaxies have not been selected for or against irregular looking morphologies.

We present here HI observations for 101 dwarf galaxies in the absolute magnitude range $-13.5>M_{r}-5 \log _{10} h_{70}>-16$ selected from the Blanton et al. (2005) catalog. These data are part of a larger, on-going survey to obtain $\mathrm{HI}$ line-widths and $\mathrm{H} \alpha$ rotation curves for a significant fraction of galaxies in this catalog with $M_{r}-5 \log _{10} h_{70}>-16$. The complete catalog contains 1034 galaxies at these luminosities. The 101 galaxies presented here are representative of the full catalog.

\subsection{Environment and the Nearest Neighbor Distances}

A goal of this paper is to understand the effects of environment on the properties of dwarf galaxies. To quantify the environment, we calculate for each dwarf galaxy the projected distance to its nearest luminous neighbor. We cannot rely on the SDSS alone to determine this distance for several reasons. First, the angular distances between nearest neighbor galaxies can be large for this nearby sample - for example, searching a $1 \mathrm{Mpc}$ region around a galaxy $30 \mathrm{Mpc}$ away corresponds to 2 degrees on the sky. Many of our dwarf galaxies are on the SDSS Southern stripes, which are only 2.5 degrees wide. In addition, because the SDSS reduction software is not optimized for large extended objects, and fails to process them correctly, the SDSS catalog does not contain many of the bright galaxies within $30 \mathrm{Mpc}$. Thus, to calculate the environments of our dwarf galaxy sample, we need a supplemental catalog that extends beyond the SDSS area and contains the brightest galaxies.

Both of these considerations drive us to use the The Third Reference Catalog of Galaxies (RC3; de Vaucouleurs et al. 1991), which is a nearly complete catalog of nearby luminous galaxies. To determine environments for our dwarf galaxies, we determine the distance to its nearest "luminous" neighbor. We define "luminous" galaxies as brighter than $M_{r}-$ $5 \log _{10} h_{70}<-19$ or have circular velocities greater than $V_{c}>100 \mathrm{~km} \mathrm{~s}^{-1}$ (Haynes et al. 1999). While we would like to include galaxies of all sizes in our environment estimate, the availability of a complete comparison catalog restricts our search to luminous neighbors. From the $B$ and $V$ photometry listed in RC3, we infer $M_{r}$ for each RC3 galaxy using the kcorrect product (see $\S 3.1$ ) . For galaxies which have the relevant entries listed, we call galaxies luminous if $M_{r}-5 \log _{10} h_{70}<-19$. While this absolute magnitude is set by the catalog limits, it roughly corresponds to the dividing line between normal and dwarf galaxies. For RC3 galaxies which do not have the relevant entries, but do have HI data listed, we call them luminous if $W_{20}>200 \mathrm{~km} \mathrm{~s}^{-1}$ ( $W_{20}$ is twice the maximum circular velocity of the HI 
gas, see $\S 2.3)$. Finally, there are some galaxies with neither HI data nor optical photometry listed in RC3. For this small set, we extract the "magnitude" from the NASA Extragalactic Database (NED), which empirically is very similar to the $B$ band RC3 magnitude, and guess $M_{r}$ based on that magnitude. Additionally, we update the coordinates in RC3 using the NED coordinates for each of the catalog objects. This set of bright galaxies is not perfectly uniform, but is suitable for our purposes.

To determine the nearest neighbor distance, we ask whether there is a luminous RC3 neighbor within a projected distance of $2.5 h_{70}^{-1} \mathrm{Mpc}$ and $300 \mathrm{~km} \mathrm{~s}^{-1}$ in redshift for each dwarf galaxy in our sample. We choose these limits because Blanton et al. (2006) have shown that environmental affects are strongest inside this scale. About $20 \%$ of our galaxies have no such neighbor; for these objects, we set the nearest neighbor distance to $2.5 \mathrm{Mpc}$. Using the SDSS Catalog Archive Server*, we have looked at wide field mosaic images around all of our objects and checked by eye that the RC3 catalog object is indeed the nearest bright neighbor. This check is not perfect due to the edge effects of SDSS, but provides a sanity check on our procedure.

\subsection{HI Observations}

We obtained HI radio observations with the Arecibo 305-m telescope and the Green Bank 100-m Telescope (GBT) in July - August 2005 and March - April 2006. At Arecibo, we used the L-wide receiver in 9-level sampling mode with 1024 channels and a $12.5 \mathrm{MHz}$ bandwidth. At GBT, we used the L-band with 8192 channels and a bandwidth of $12.5 \mathrm{MHz}$. The Arecibo configuration yields a velocity resolution of $2.5 \mathrm{~km} \mathrm{~s}^{-1}$, the GBT configuration yields a resolution of $0.3 \mathrm{~km} \mathrm{~s}^{-1}$. Standard ON/OFF position switching was used during both the Arecibo and GBT observations. The total on-source integration times were on average 10 minutes for the Arecibo data and 20 minutes for the GBT data. The average half-light radius of the dwarf galaxies in our sample is $\sim 8^{\prime \prime}$ and they should be completely contained within the radio beamsize of $3^{\prime}$ and $9^{\prime}$ for Arecibo and GBT, respectively. We

have searched the beam coverage for nearby contaminating galaxies and have removed the few cases in which a nearby galaxy is contained in the beam and is within $100 \mathrm{~km} \mathrm{~s}^{-1}$ of the targeted galaxy. Optical images and HI profiles for three representative galaxies are shown in Figure 1. Our observations yielded strong HI detections for 88 of 101 galaxies. The 13 galaxies which did not yield HI detections are represented by upper limits in the analysis of $\S 3.1$ and are excluded from the Tully-Fisher analysis in $\S 3.2$, since we cannot estimate HI

${ }^{*}$ http: //cas.sdss.org 
line-widths for these galaxies.

We compute the $20 \% \mathrm{HI}$ line-width (W20) by finding the peak HI flux within $150 \mathrm{~km} \mathrm{~s}^{-1}$ of the optical radial velocity of each galaxy and computing the difference between the nearest points having $20 \%$ of the peak flux. The integrated HI flux is calculated by expanding the W20 values by $20 \mathrm{~km} \mathrm{~s}^{-1}$ on a side and integrating the flux in this region. Errors bars on the line widths and integrated fluxes were computed using a Monte Carlo bootstrap method: noise was added to the stacked one-dimensional radio spectra based on the observed variance in the baseline and the observed quantities measured. Error bars on the line-width and integrated flux were calculated from the scatter in the mean quantities recovered form the Monte Carlo simulations. We convert the HI integrated flux into HI mass based on the optically-thin approximation: $M_{\mathrm{HI}}=2.356 \times 10^{5} D^{2} F_{\mathrm{HI}}$ where $\mathrm{D}$ is the distance in $\mathrm{Mpc}$ and $F_{\mathrm{HI}}$ is the integrated HI flux in Jy $\mathrm{km} \mathrm{s}^{-1}$ (Eqn. 9; Haynes \& Giovanelli 1984). We note that this standard HI mass conversion is a lower limit to the true HI mass if the galaxy contains optically thick HI. Error bars on the HI mass include the HI flux error and the error in the distance.

\section{Results}

The 101 dwarf galaxies presented in this paper represent the largest homogeneous sample of galaxies fainter than $M_{r}-5 \log _{10} h_{70}>-16$ with well-measured HI and optical properties. Properties of the sample are listed in Table 1; the distribution of these properties is shown in Figure 2. The average absolute magnitude, corrected for Galactic extinction (Schlegel et al. 1998), of our sample is $\left\langle M_{r}\right\rangle=-15.4$ at an average distance of $30 h_{70}^{-1} \mathrm{Mpc}$. Galaxies in the sample tend to be blue, $\langle g-r\rangle=0.3$, reflecting the fact that our sample does not probe the densest environments where red dwarf ellipticals tend to reside. The galaxies have an average effective surface brightness of $\left\langle\mu_{r, \text { eff }}\right\rangle=22.4 \mathrm{mag} \operatorname{arcsec}^{-2}$, and exponential surface brightness profiles as measured by the Sérsic index $\left(\left\langle n_{\text {Sersic }}\right\rangle=1.1\right)$. The average effective half-light $r$-band radius is $\left\langle r_{\text {eff }, r}\right\rangle=8^{\prime \prime}=1.0 h_{70}^{-1} \mathrm{kpc}$. These galaxies exist in environments ranging from dense groups to truly isolated objects: about $15 \%$ of the sample are satellites of luminous host galaxies, defined as being within $200 h_{70}^{-1} \mathrm{kpc}$ projected distance and 300 $\mathrm{km} \mathrm{s}^{-1}$ in velocity of a galaxies with $M_{r}-5 \log _{10} h_{70}<-19$ (see $\S 2.2$ ). Meanwhile, about $65 \%$ of the galaxies are further than $500 h_{70}^{-1} \mathrm{kpc}$ of any such host galaxy - they are very isolated galaxies.

The dwarf galaxies in our sample were not selected with respect to morphology or gas content. The sample consists of $\sim 10 \%$ gas-poor dwarf galaxies commonly known as "dwarf spheroidal" or "dwarf elliptical" galaxies. The majority of our sample consists of gas-rich 
dwarfs commonly known as "dwarf irregular" galaxies. This latter term is commonly misinterpreted to mean that gas-rich dwarf galaxies have disorganized morphology or turbulent kinematics. Historically, the term "irregular" indicates that the HII regions in a galaxy do not follow a regular spiral arm pattern as observed in normal massive disk galaxies (Sandage 1975; Skillman 1996). Indeed, our sample dwarf galaxies do not show any evidence of spiral structure, based on the visual inspection of unsharp-masked SDSS $r$-band images. However, our dwarfs do have well-defined optical centers and regular morphology (Figure 1) as has been noted by previous studies of dwarf galaxies in this regime (Matthews \& Gallagher 2002). Our HI radio profile shapes suggest that our dwarf galaxies have regular rotation fields: $18 \%$ of the HI profiles have distinct double-peaked shapes implying a coherent rotation field; this fraction increases to $30 \%$ if we limit the sample to edge-on galaxies $(b / a<0.4)$ only. Thus while the classification "dwarf irregular" is correct for the majority of our sample galaxies, due to its common misinterpretation, we simply refer to all the galaxies in this sample simply as low luminosity dwarf galaxies.

\subsection{HI Gas Fractions}

We first investigate the ratio of gas mass to total baryonic mass in our dwarf galaxy sample. We define the gas fraction as $f_{\text {gas }}=M_{\text {gas }} /\left(M_{\text {gas }}+M_{\text {stellar }}\right)$. The denominator of this expression is the total baryonic mass defined as the sum of the gas and stellar mass. We take $M_{\text {gas }}=1.4 M_{\mathrm{HI}}$, where $M_{\mathrm{HI}}$ is calculated in $\S 2.3$ and the multiplicative factor takes in account the presence of helium and metals. We do not include a correction term for molecular hydrogen $\left(\mathrm{H}_{2}\right)$ as the $\mathrm{H}_{2} / \mathrm{HI}$ ratio is much lower in dwarf galaxies compared to luminous spirals (Leroy et al. 2005; Taylor et al. 1998). This deficiency holds even after accounting for a strong metallicity dependence of the $\mathrm{H}_{2}$ tracer molecule CO (Garnett 2002). The stellar mass, $M_{\text {stellar }}$, is based on the optical SDSS $i$-band magnitude and $(g-r)$ colors using the mass-to-light ratios determined from the galaxy evolution models of Bell et al. (2003) using the Kroupa et al. (1993) initial mass function (that is, we subtract 0.15 dex from Bell et al. Table 7 calculated for a 'diet' Salpeter initial mass function). We choose this combination of magnitude and color to minimize contamination from emission lines and thus probe the "true" stellar continuum (West 2005). We have calculated the stellar mass based on the broad-band fluxes using the kcorrect product ${ }^{\dagger}$ and based on the spectra published by Kauffmann et al. (2003). Both these methods produce somewhat lower stellar mass $(\sim 30 \%)$ than predicted by Bell et al. We stress that the mass-to-light ratios are very sensitive to the assumed initial mass function. We note that the gas fraction does not depend on the

\footnotetext{
${ }^{\dagger}$ http: //cosmo.nyu.edu/blanton/kcorrect; Blanton et al. (2003)
} 
distance to individual galaxies.

The average gas fraction for our sample is $\left\langle f_{\text {gas }}\right\rangle=0.6$, with gas fractions as high as $95 \%$. Thus, for the majority of the dwarf galaxies in our sample, the baryonic mass is dominated by gas, rather than stars. In Figure 3 we plot gas fraction as a function of absolute $r$-band magnitude. The flux limits of our sample are clearly seen in this figure. We compare our results to representative samples from the literature by converting the literature magnitudes to $r$-band using the photometric transformations from kcorrect (see the web page referenced above) and recalculate gas fractions using the same methods described above. The most comparable sample of galaxies is that of LSB dwarf galaxies by Schombert, McGaugh, \& Eder (2001). The gas fractions in this sample are comparable to our SDSS (higher surface brightness) sample. For more luminous spiral galaxies, the average gas fraction is much lower: Bell \& de Jong (2000) find an average gas fraction of $30 \%$ for a sample of low-inclination spiral galaxies. The average gas fraction for the large field spiral sample of Haynes et al. (1999) is 25\%, assuming an average color $V-I=1.0$. A clear trend is seen in Figure 3 : less luminous galaxies tend toward higher gas fractions. While galaxies exist in the lower left region of the plot (faint, gas-poor dwarf elliptical or dwarf spheroidal galaxies), they are rare in the upper right region (based on the HIPASS sample, West et al. in prep). In other words, galaxies fainter than $M_{r}-5 \log _{10} h_{70} \sim-16$ span the full range of possible gas fractions, while there is an upper limit to the gas fractions of brighter galaxies which depends on absolute magnitude. This trend has been noted by several authors (McGaugh \& de Blok 1997; Schombert et al. 2001; Lee et al. 2002); Figure 3 extends this to significantly fainter absolute magnitudes compared to previous studies.

We interpret the gas fractions in Figure 3 as evidence that, compared to more luminous galaxies, dwarf galaxies are far less efficient at turning gas into stars over their lifetimes. This is likely related to lower on average gas surface densities in lower luminosity systems. Kennicutt (1998), Martin \& Kennicutt (2001) and references therein have shown for luminous galaxies that star formation does not occur below a critical gas density $\left(\sim 5 M_{\odot} \mathrm{pc}^{-2}\right)$. Hunter et al. (1998) confirm that this limit holds for low mass systems, although the critical density is slightly lower $\left(\sim 3 M_{\odot} \mathrm{pc}^{-2}\right)$, due in part to the lack of spiral arms and solid-body kinematics in the inner regions of dwarfs. We cannot precisely measure the average gas density in this sample because our HI single-dish observations do not provide an estimate of the HI radius. However, we can estimate the HI radius by assuming it scales (roughly) with the optical radius. We adopt an average HI-to-optical radius scaling calculated by Begum et al. (2006) of $r_{\mathrm{HI}} / r_{\mathrm{H}_{\alpha}}=2.5$. The average gas density of our sample is $1.5 M_{\odot} \mathrm{pc}^{-2}$ with more than a factor of two scatter. Thus, the majority of galaxies in this sample are at, or below, the critical gas density required for star formation. If the threshold described in Kennicutt (1998) exists, the star formation in these dwarf galaxies implies that the local density of gas 
in the regions of star formation significantly exceeds the global density averaged over the whole galaxy.

In the top right panel of Figure 4, we show that the gas fraction is a strong function of $g-r$ color in the sense that bluer galaxies in our sample are gas-rich. We assume that the spread in $g-r$ color represents a spread in time-averaged star formation over the past 1 Gyr, as opposed to a spread in metallicity, for two reasons. First, the bluest colors in our sample cannot be explained by old, metal poor stellar population synthesis models. Second, the majority of galaxies in our sample are currently forming stars (as indicated by $\mathrm{H} \alpha$ emission in the SDSS spectra) which dominates their broad-band colors (Bruzual \& Charlot 2003). Thus, in the top left of Figure 4, the gas-rich galaxies have had a higher fraction of their star-formation in the recent past. In contrast, the current star formation rate per unit area (within the last few million years) is not correlated with gas fraction (Figure 4, bottom left). We calculate this current star formation rate based on the $u$-band flux using the conversion from Hopkins et al. (2003). For comparison, we also calculated the star formation rates based on the $\mathrm{H} \alpha$ emission line flux in the SDSS spectra (detected for most of our galaxies), but found that the resulting rates suffered from the uncertainty of extrapolating the $\mathrm{H} \alpha$ flux from the $3^{\prime \prime}$ SDSS fiber aperture. We normalize the total star formation rate by the area over which star formation is occurring in order to remove any size-dependent variations within our sample. The median total star formation rate per unit area based on the $u$-band for our sample is $S F R=1 \times 10^{-3} M_{\odot} \mathrm{yr}^{-1} \mathrm{kpc}^{-2}$; These rates are comparable to previous studies of gas-rich galaxies in this luminosity range (Hunter \& Elmegreen 2004; van Zee 2001; Barazza et al. 2006). The star formation rates per unit area are shown in the bottom left of Figure 4 and are not correlated with gas fraction. The gas fraction is better correlated with the time-averaged star-formation rate over the last billion years $(g-r$ color $)$ than with the recent star-formation rate in the last few million years. This implies that the star formation rates we are observing now are different than those in the recent past; the star formation rates appear to be stochastic.

We attempt to quantify the stochastic nature of star formation in our sample by calculating the stellar birthrate timescale. In the left panel of Figure 5, we plot the observed stellar mass divided by the current star formation rate $\left(M_{\text {stellar }} / S F R_{\text {tot }}\right)$. If the current rate of star formation has been constant over the lifetime of a galaxy, this quantity would represent the time needed to form its observed stellar component. For our sample, the median timescale to make stars is 15 Gyrs with a nearly symmetric scatter to both shorter and longer timescales. Observations of the color-magnitude diagrams of dwarf galaxies in the Local Group suggest that all dwarfs have an ancient stellar population component (Mateo 1998; Dolphin et al. 2005). If we assume all the galaxies in our sample formed at early times (10-12 Gyr), this implies that roughly half the sample had lower star formation rates and half had higher rates 
in the past than presently observed. This line of argument again suggests that star formation in dwarfs fluctuates and that the current star formation rate is not necessarily representative of the time-averaged rate. In the right panel of Figure 5 we also investigate the future of star formation by plotting the gas consumption timescale $\left(M_{\text {gas }} / S F R_{\text {tot }}\right)$. In this plot we have not included gas-recycling, which would increase the gas consumption timescales. The majority of galaxies in this sample will be able to make stars, and therefore consume gas, at their present rates for another Hubble time.

The strongest observed trend in our sample is that between the gas fraction and $g-r$ color (Figure 4, top left panel). This trend may be due to either internal or external processes. For example, a galaxy may use up its gas by forming stars and thus have redder colors due to a lower star formation rate compared to the recent past. Alternatively, a galaxy's gas could be removed in denser environments and appear redder due to quenching of its star formation. Since we have quantified the environment of each galaxy (see $\S 2.2$ ), we are able to test whether this and other trends are due internal or external processes.

The top right panel of Figure 4 suggests that both internal and external processes are important in controlling the trend between gas fraction and color. For gas-rich galaxies in our sample, with $f_{\text {gas }}>0.4$, the gas fractions are uncorrelated with nearest neighbor distance. In contrast, galaxies with low gas fractions $\left(f_{\text {gas }}<0.4\right)$ are preferentially found within $0.5 \mathrm{Mpc}$ of a luminous neighbor galaxy. Thus, it appears that some reduction in the gas fraction is related to internal processes such as normal star-formation, "primordial" removal of gas at reionization, or outflows, however, only environmental effects are very effective at removing all of the gas from a dwarf galaxy. This is further supported by the bottom right panel of Figure 4. While red galaxies only exist near luminous neighbors, the colors of the blue sequence galaxies do not depend on distance to the nearest neighbor. This result is similar to the relationship between color and environment, or morphology and environment, seen in more luminous galaxies (e.g. in Hubble 1936; Oemler 1974; Norberg et al. 2002; Blanton et al. 2005a), and the dwarf galaxy population of the Local Group (Grebel et al. 2003). We conclude that while internal processes are able to reduce the gas fractions to $f_{\text {gas }} \sim 0.4$, external processes are require to lower the gas fractions (via removal of gas as suggested below via residuals in the Tully-Fisher relation) further.

\subsection{The Baryonic Tully-Fisher Relationship}

The Tully-Fisher relationship relates the mass of a galaxy as inferred from its luminous component to its dynamical mass, as measured by the maximum rotational velocity. The tight coupling between these components has greatly informed our understanding of galaxy 
formation (Mo et al. 1998; van den Bosch 2000; Bullock et al. 2001; Mayer \& Moore 2004). Dwarf galaxies with rotation velocities less than $90 \mathrm{~km} \mathrm{~s}^{-1}$ tend to fall below the optical Tully-Fisher relationship established by massive galaxies in the sense that the ratio of stellar to dynamical mass is lower for the dwarfs (McGaugh et al. 2000; Matthews et al. 1998). This "break" has sometimes been interpreted as evidence that dwarf galaxies have preferentially lost baryonic mass due to e.g. supernovae winds (Dekel \& Silk 1986; Dekel \& Woo 2003). McGaugh et al. (2000) first noted that this break is removed when both the stellar and gaseous components are taken into account. This baryonic Tully-Fisher relationship has been investigated by many groups (Bell \& de Jong 2001; Verheijen 2001; Gurovich et al. 2004; McGaugh 2005). Understanding the baryonic Tully-Fisher relation for low mass galaxies should place tight constraints on the role of ram pressure stripping, tidal stripping, feedback, galactic winds, photoevaporation during reionization in the evolution of dwarf galaxies.

In Figures 6 and 7, we explore the Tully-Fisher relationship within our dwarf galaxy sample. We estimate the maximum rotational velocities for our galaxies based on the inclinationand turbulence-corrected $\mathrm{HI}$ profile half-width $\left(\mathrm{W} 20_{i, t} / 2\right)$. While we will present resolved optical $\mathrm{H} \alpha$ rotation curves for this sample in a forthcoming paper, the HI line-widths are a more reliable measure of the maximum rotation velocity in these low mass systems. This is because the optical rotation curves of low mass galaxies rarely show a velocity turnover at the last optically measured data point (Matthews \& Gallagher 2002), leading to an underestimate of the true maximum rotation velocity. Resolved HI rotation curves also do not always show a velocity turnover (McGaugh et al. 2001), however the HI tends to extend 2-3 times beyond the observed optical radii (Begum et al. 2006) and thus is a more reliable probe of the true maximum rotation velocity.

The dwarf galaxies in our sample have a significant rotation component (Figure 1). We correct the observed HI line-widths for line broadening due to turbulent velocity dispersion and inclination using the formula first proposed by Bottinelli et al. (1983):

$$
\mathrm{W} 20_{i, t}=\frac{\mathrm{W} 20-\mathrm{W} 20_{t}}{\sin i}
$$

Where W20 is the observed HI line-width, W20 is the turbulent velocity correction term and $i$ is the inclination angle inferred from the optical images. We confirm the validity of a linear turbulence correction based on modeling the integrated velocity profiles of simulated galaxies constructed from Hernquist (1993) model disk galaxies. For nearby dwarf galaxies with rotation velocities similar to our sample, Begum et al. (2006) have measured velocity dispersion in the gas component of $\sigma_{\mathrm{los}}=8 \mathrm{~km} \mathrm{~s}^{-1}$ based on the average line-of-sight velocity dispersion measured from $2 \mathrm{D}$ velocity maps. Using the Begum et al. value, this results in a turbulence correction of $\mathrm{W} 20_{t}=16 \mathrm{~km} \mathrm{~s}^{-1}$, which we use here. Altering this turbulence correction (say to $25 \mathrm{~km} \mathrm{~s}^{-1}$ ) does not change our results below. 
Using the approach of Haynes \& Giovanelli (1984), we infer $\sin i$ from the $b / a$ axis ratio based on two-dimensional Sérsic fitting of the $r$-band SDSS images. In particular, we assume the disks to be intrinsically oblate and axisymmetric, with a three-dimensional axis ratio of $q_{0}$, and take:

$$
\sin i=\sqrt{\frac{1-(b / a)^{2}}{1-q_{0}^{2}}},
$$

with $q_{0}=0.19$. Again, changing the particular value of $q_{0}$ (say to 0.3 ) again does not change our results significantly.

Figure 6 shows the stellar, HI and baryonic Tully-Fisher relationships for our dwarf galaxy sample. In the three panels of this figure, we linearly regress to fit a function of the form:

$$
\log _{10}\left(\frac{\mathrm{W} 20_{i, t} / 2}{50 \mathrm{kms}^{-1}}\right)=a+b \log _{10}\left(\frac{\text { mass }}{10^{8} h_{70}^{-2} M_{\odot}}\right)
$$

where $a$ and $b$ are the fitted intercept and slope of the Tully-Fisher relationship, respectively. These fits are regressions with mass as the independent variable. Table 2 lists their values and uncertainties (calculated from bootstrap resampling). In addition, we calculate the correlation coefficient $r$ between each pair of variables, listed in each panel of Figure 6 . The correlation coefficient is zero for two unrelated quantities and unity for two perfectly linearly related quantities (e.g. Lupton 1993).

The stellar Tully-Fisher (Figure 6, right panel) has significantly more scatter than the either the HI or total baryonic relationships (Figure 6, middle and left panels). The stellar relation has a much lower correlation coefficient $(r \sim 0.3$ versus $r \sim 0.8)$ and considerably more scatter about the best fit regression line, as listed in Table 2 (about $50 \%$ scatter compared to about $30 \%$ scatter), than do the HI mass or baryonic mass TF relationships. This result is perhaps not surprising: at low luminosities, stars contribute far less to the total baryonic mass compared to massive galaxies (Figure 3). Thus, for dwarf galaxies of the same total mass, the amount of gas turned into stars varies between systems. While the scatter in the baryonic Tully-Fisher relationship is much smaller compared to the stellar relationship, it does have more scatter than accounted for by our observational error bars. The fitted slope is unchanged if we restrict our sample to only galaxies whose inclination corrections are small $(b / a<0.4)$ or if we alter our assumptions about turbulence or the intrinsic axis-ratio.

In Figure 7, we compare our dwarf galaxy sample to Tully-Fisher data available in the literature. We compare only to literature samples for which HI and optical measurements are available: Haynes et al. (1999), Verheijen (2001), Matthews et al. (1998), and McGaugh et al. (2000). For the literature datasets, we recalculate distances based on $H_{0}=70 \mathrm{Mpc}$ 
$\mathrm{km} \mathrm{s}^{-1}$ and a model of the local velocity field (Willick et al. 1997) (for galaxies with recession velocities less than $6000 \mathrm{~km} \mathrm{~s}^{-1}$ ). For the Haynes et al. (1999) data, we assume a constant color of $V-I=1.0$.

In the left panel of Figure 7 we plot the optical Tully-Fisher relationship in the $I$-band. McGaugh et al. (2000) first noted that low mass dwarf galaxies fall well below the optical Tully-Fisher (TF) relationship as defined by luminous spiral galaxies in the sense that, for a given rotation velocity, the dwarfs are optically under-luminous compared to the expected TF value. McGaugh et al. also noted that these same galaxies lie on the same relation defined by luminous galaxies in the baryonic TF relationship (Figure 7, right panel). Again, this is not surprising: we have shown above that dwarf galaxies have high gas fractions and the optical luminosity/stellar mass is often only a few percent of the total baryonic mass. However, unlike McGaugh et al., we do not see evidence for a distinct break in slope of the optical TF occurring at $90 \mathrm{~km} \mathrm{~s}^{-1}$. At low luminosities, the scatter around the optical TF relation does increase considerably, but not preferentially to high or low velocities.

That a single linear fit adequately describes the baryonic TF data over more than three decades of mass is significant. Many authors have suggested that supernovae-driven winds will preferentially remove baryonic material from galaxies with $v_{\max }<100 \mathrm{~km} \mathrm{~s}^{-1}$ due to their far smaller gravitational potentials compared to luminous galaxies (Dekel \& Silk 1986; Dekel \& Woo 2003). Figure 7 suggests that a critical mass threshold does not exist below which mass-ejection is efficient down to baryonic masses of $10^{8} M_{\odot}$ and rotational velocities of as low as $20 \mathrm{~km} \mathrm{~s}^{-1}$. If a critical threshold existed, we would have expected to see a break in the baryonic Tully-Fisher.

While it might be possible to explain this single relationship via a baryonic mass-loss rate which varies smoothly with mass, the measured slope of this relation suggests that this is not the case. From the virial theorem, the expected slope of the baryonic TF is $b=0.33$. The predicted slope from cosmological simulations is $b \sim 0.29$, or as more commonly quoted $1 / b \sim 3.5$ (Bullock et al. 2001; Kravtsov et al. 2004), in good agreement with our value of $b=0.27 \pm 0.01$, or $1 / b=3.70 \pm 0.15$. Thus, we interpret the baryonic TF relationship shown in Figure 7 as evidence that dwarf galaxies do not preferentially lose significant baryonic mass compared to luminous galaxies.

Finally, we consider the residuals from the TF relationship. We define these residuals in terms of the ratio $W 20_{i, t} / W 20_{i, t}(T F)$, the corrected observed HI line-width divided by that expected from the fitted linear baryonic Tully-Fisher relationship. This quantity is defined in the sense that larger $W 20_{i, t} / W 20_{i, t}(T F)$ values correspond to lower inferred baryon-to-dark matter ratios. Figure 8 shows this quantity as a function of projected nearest neighbor distance, color, gas fraction and axis ratio $b / a$. We find no correlation between 
these residuals and the axis-ratio $b / a$, except that the very flat galaxies $(b / a<0.25)$ never exhibit low $W 20_{i, t} / W 20_{i, t}(T F)$ ratios (Figure 8, bottom left panel). That no strong trend exists with axis-ratio suggests that the measured HI line-widths are properly sampling the galactic potential. Dwarf galaxies with low gas fractions have low $W 20_{i, t} / W 20_{i, t}(T F)$ ratios. This suggests that low gas fraction dwarf galaxies, which are preferentially found in dense environments, have higher baryon-to-dark matter ratios compared to the majority of dwarfs in our sample. If, for example, reduced gas fractions in dense environments were due to interaction-induced star formation, we would expect these ratios to remain constant. If this were due to ram pressure stripping alone one might expect this ratio to be lower in dense environments: gas stripping would preferentially remove baryonic mass, reducing the ratio of baryons to dark matter. There is little correlation with color, indicating that if gas stripping is important, it is not directly effecting to the star-formation rates at the center. Alternatively, it is possible that this is simply a selection effect and the HI line-widths do not properly sample the galactic potentials of the lowest gas fraction galaxies. As the number of observed galaxies in this mass range increase, the $\mathrm{TF}$ residuals should place significant constraints on galaxy evolution processes important at these masses.

\section{Conclusions}

We present results from an HI survey of low luminosity dwarf galaxies in the absolute magnitude range $-13.5>M_{r}-5 \log _{10} h_{70}>-16$ selected from the SDSS. These data represent the largest homogeneous sample of low luminosity dwarf galaxies with well-measured HI and optical properties. The sample spans a range of total baryonic masses between $\mathrm{M}_{\text {baryonic }}=10^{8}-10^{9} M_{\odot}$ with measured HI line-widths between $\left\langle v_{\text {rot }}\right\rangle=\left\langle\mathrm{W} 20_{i, t} / 2\right\rangle=20-80$ $\mathrm{km} \mathrm{s}^{-1}$. The dwarf galaxies in this sample are found in a wide range of environments, from dense groups to truly isolated galaxies.

For the majority of our dwarf galaxy sample, the total galactic baryonic mass is dominated by the gas mass rather than stellar mass. The average gas fraction for our sample is $\left\langle f_{\text {gas }}\right\rangle=0.6$, with gas fractions as high as $95 \%$. This significantly exceeds that of gas-rich galaxies at higher luminosities. Thus, dwarf galaxies are inefficient at turning gas into stars compared to more luminous galaxies. We find that gas-rich galaxies have bluer $g-r$ colors compared to gas-poor objects implying that gas-rich dwarf galaxies have had more recent star formation. The majority of galaxies in our sample are currently forming stars based on observed $\mathrm{H} \alpha$ emission in the SDSS spectra. We find that the current rate of star formation

per unit area (based on the total $u$-band flux) is not correlated with gas fraction, confirming previous results suggesting that the star formation rates in dwarf galaxies are stochastic (van 
Zee 2001; Hunter \& Elmegreen 2004). Thus, dwarf galaxies form stars with a rate that varies over million year time scales, but is correlated with the gas fraction over longer time periods.

Using the range of environments within our sample, we examine whether internal or external processes control the gas fractions of dwarf galaxies. Trends between galaxy properties, such as color or morphology, and environment have been well studied for galaxies at brighter luminosities (Norberg et al. 2002; Blanton et al. 2005a), but have been previously limited to the Local Group and nearby groups at dwarf luminosities (Grebel et al. 2003). Our sample allows us to quantify these trends for dwarf galaxies over a much wider range of environments. We find that dwarf galaxies with gas fractions $f_{\text {gas }}<0.4$, comprising $15 \%$ of this sample, are exclusively found within $0.5 \mathrm{Mpc}$ of a luminous neighbor galaxy. Galaxies with gas fractions $f_{\text {gas }}>0.4$ are found across the full range of environments contained within this sample. Thus, while internal galaxies processes can reduce the gas fractions, external processes are required to fully remove gas from a dwarf galaxy.

The optical Tully-Fisher relationship within our dwarf galaxy sample demonstrates considerable scatter. As previous work has shown for brighter samples (Bell \& de Jong 2001; Verheijen 2001; Gurovich et al. 2004; McGaugh 2005), the baryonic Tully-Fisher relationship within our sample is much tighter. When we consider the Tully-Fisher relationship across all absolute magnitudes, we find a logarithmic slope of $b=0.27 \pm 0.01$ ( or $1 / b=3.70 \pm 0.15$ ). These results are in accord with CDM predictions of $1 / b \sim 3.5$ assuming no preferential loss of baryons as a function of mass (Bullock et al. 2001). Thus, our results suggest that processes which preferentially remove gas from dwarf galaxies are not important for the majority of galaxies in our sample with maximum rotation velocities $v_{\max } \sim 20-80 \mathrm{~km} \mathrm{~s}^{-1}$ (roughly corresponding to total dynamical masses of $\mathrm{M}_{\text {total }}=10^{9}-10^{10} M_{\odot}$ ).

If galaxies are associated one-to-one with $\Lambda$ CDM dark matter halos, the mass-to-light ratios of galaxies must decline as a function of host halo mass (Benson et al. 2003). The most well-known example of this problem is the Local Group "substructure problem" (Klypin et al. 1999; Moore et al. 1999). To explain this trend in mass-to-light ratio, investigators often invoke processes such as ram pressure stripping or supernova feedback that either removes gas or heats it sufficiently to prevent star-formation in low mass galaxies (e.g., Dekel \& Silk 1986; Dekel \& Woo 2003; Mori \& Burkert 2000; Bullock et al. 2000). Most semi-analytic models require significant baryonic mass loss (Cole et al. 2000; Benson et al. 2003; Croton et al. 2005), predicting baryon-to-dark matter ratios for dwarf galaxies well below the "Universal" baryon fraction (Read \& Trentham 2005). Comparing the metallicity of galaxies over a wide range of baryonic masses, Tremonti et al. (2004) found that dwarfs are metal depleted relative to massive galaxies and argue in favor of galactic winds efficiently removing enriched material from low mass galaxies. However, at the masses of the galaxies in our sample, 
detailed models of the physics of gas blow-out and blow-away tend to disfavor significant gas mass loss, predicting loss of only a few percent (Mac Low \& Ferrara 1999; Ferrara \& Tolstoy 2000). Our sample cannot rule out small amounts baryon mass loss from these galaxies, nor scenarios in which gas remains bound to the galaxy but is blown away from the region participating in star formation. This may explain the low observed metallicities of dwarf galaxies - enriched gas is removed from regions of active star formation, resulting in low observed metallicities, but either remains bound or represents only a few percent of the total baryonic mass. Indeed, recent cosmological simulations which include realistic feedback and chemical evolution suggest that the metallicity, mass-to-light ratios and other galaxies properties can be explained without including supernova energy feedback (Tassis et al. 2006). Our results favor such dwarf galaxy formation models in which processes such as supernovae winds are not responsible for significant gas or baryon mass in low mass galaxies.

We thank David Weinberg and Rob Kennicutt for early and important encouragement on this project. We had very productive conversations with many people regarding this paper including Andrey Kravtsov, Jeremy Darling, Oleg Gnedin, Evan Skillman, Beth Willman, Jay Gallagher, Ari Maller, Kathryn Johnston, David W. Hogg, and Stéphane Courteau. We would like to thank Carl Bignell, Toney Minter, and Karen O'Neil for help with the GBT observations. We thank Martin Kirby for creative assistance in naming the project. M. G. is supported by NASA through Hubble Fellowship grant HF-01159.01-A awarded by the Space Telescope Science Institute, which is operated by the Association of Universities for Research in Astronomy. M. M. is supported by NASA NAG5-11669 and NSF AST-0428465. A. W. is supported by NSF AST-0540567.

\section{REFERENCES}

Abazajian, K. et al. 2004, AJ, 128, 502

Barazza, F. D., Jogee, S., Rix, H.-W., Barden, M., Bell, E. F., Caldwell, J. A. R., McIntosh, D. H., Meisenheimer, K., Peng, C. Y., \& Wolf, C. 2006, ApJ, 643, 162

Begum, A., Chengalur, J. N., Karachentsev, I. D., Kaisin, S. S., \& Sharina, M. E. 2006, MNRAS, 365, 1220

Bell, E. F. \& de Jong, R. S. 2000, MNRAS, 312, 497

Bell, E. F. \& de Jong, R. S. 2001, ApJ, 550, 212

Bell, E. F., McIntosh, D. H., Katz, N., \& Weinberg, M. D. 2003, ApJS, 149, 289 
Benson, A. J., Bower, R. G., Frenk, C. S., Lacey, C. G., Baugh, C. M., \& Cole, S. 2003, ApJ, 599, 38

Binggeli, B., Sandage, A., \& Tammann, G. A. 1985, AJ, 90, 1681

Blanton, M. R., Brinkmann, J., Csabai, I., Doi, M., Eisenstein, D. J., Fukugita, M., Gunn, J. E., Hogg, D. W., \& Schlegel, D. J. 2003, AJ, 125, 2348

Blanton, M. R., Eisenstein, D., Hogg, D. W., Schlegel, D. J., \& Brinkmann, J. 2005a, ApJ, 629,143

Blanton, M. R. et al. 2005b, AJ, 129, 2562

Blanton, M. R. et al. 2005, ApJ, 631, 208

Blanton, M. R. et al. 2006, ApJ, in press (astro-ph/0411037)

Bottinelli, L., Gouguenheim, L., Paturel, G., \& de Vaucouleurs, G. 1983, A\&A, 118, 4

Bruzual, A. G. \& Charlot, S. 2003, MNRAS, 344, 1000

Bullock, J. S., Dekel, A., Kolatt, T. S., Kravtsov, A. V., Klypin, A. A., Porciani, C., \& Primack, J. R. 2001, ApJ, 555, 240

Bullock, J. S., Kravtsov, A. V., \& Weinberg, D. H. 2000, ApJ, 539, 517

Cole, S., Lacey, C. G., Baugh, C. M., \& Frenk, C. S. 2000, MNRAS, 319, 168

Croton, D. J. et al. 2005, MNRAS, submitted (astro-ph/0508046)

de Vaucouleurs, G., de Vaucouleurs, A., Corwin, H. G., Buta, R. J., Paturel, G., \& Fouque, P. 1991, Third Reference Catalogue of Bright Galaxies (Volume 1-3, XII, 2069 pp. 7 figs.. Springer-Verlag Berlin Heidelberg New York)

Dekel, A. \& Silk, J. 1986, ApJ, 303, 39

Dekel, A. \& Woo, J. 2003, MNRAS, 344, 1131

Dolphin, A. E., Weisz, D. R., Skillman, E. D., \& Holtzman, J. A. 2005, astro-ph/0506430

Ferguson, H. C. \& Sandage, A. 1991, AJ, 101, 765

Ferrara, A. \& Tolstoy, E. 2000, MNRAS, 313, 291

Garnett, D. R. 2002, ApJ, 581, 1019 
Giovanelli, R. et al. 2005, AJ, 130, 2598

Gnedin, N. Y. 2000, ApJ, 535, 530

Grebel, E. K., Gallagher, III, J. S., \& Harbeck, D. 2003, AJ, 125, 1926

Gurovich, S., McGaugh, S. S., Freeman, K. C., Jerjen, H., Staveley-Smith, L., \& De Blok, W. J. G. 2004, Publications of the Astronomical Society of Australia, 21, 412

Gutiérrez, C. M., Azzaro, M., \& Prada, F. 2002, ApJS, 141, 61

Haynes, M. P. \& Giovanelli, R. 1984, AJ, 89, 758

Haynes, M. P., Giovanelli, R., Chamaraux, P., da Costa, L. N., Freudling, W., Salzer, J. J., \& Wegner, G. 1999, AJ, 117, 2039

Hernquist, L. 1993, ApJS, 86, 389

Hopkins, A. M., Miller, C. J., Nichol, R. C., Connolly, A. J., Bernardi, M., Gómez, P. L., Goto, T., Tremonti, C. A., Brinkmann, J., Ivezić, Ž., \& Lamb, D. Q. 2003, ApJ, 599, 971

Hubble, E. P. 1936, The Realm of the Nebulae (New Haven: Yale University Press)

Hunter, D. A. \& Elmegreen, B. G. 2004, AJ, 128, 2170

Hunter, D. A., Elmegreen, B. G., \& Baker, A. L. 1998, ApJ, 493, 595

Impey, C. D., Sprayberry, D., Irwin, M. J., \& Bothun, G. D. 1996, ApJS, 105, 209

Karachentsev, I. D., Karachentseva, V. E., Huchtmeier, W. K., \& Makarov, D. I. 2004, AJ, 127,2031

Kauffmann, G. et al. 2003, MNRAS, 341, 33

Kennicutt, R. C. 1998, ARA\&A, 36, 189

Klypin, A., Gottlöber, S., Kravtsov, A. V., \& Khokhlov, A. M. 1999, ApJ, 516, 530

Kravtsov, A. V., Gnedin, O. Y., \& Klypin, A. A. 2004, ApJ, 609, 482

Kroupa, P., Tout, C. A., \& Gilmore, G. 1993, MNRAS, 262, 545

Lee, H. et al. 2006, ApJ, in press, (astro-ph/0605036)

Lee, J. C., Salzer, J. J., Impey, C., Thuan, T. X., \& Gronwall, C. 2002, AJ, 124, 3088 
Leroy, A., Bolatto, A. D., Simon, J. D., \& Blitz, L. 2005, ApJ, 625, 763

Lupton, R. 1993, Statistics in theory and practice (Princeton, N.J.: Princeton University Press, - c1993)

Mac Low, M.-M. \& Ferrara, A. 1999, ApJ, 513, 142

Martin, C. L. \& Kennicutt, R. C. 2001, ApJ, 555, 301

Mateo, M. L. 1998, ARA\&A, 36, 435

Matthews, L. D. \& Gallagher, J. S. 2002, ApJS, 141, 429

Matthews, L. D., van Driel, W., \& Gallagher, J. S. 1998, AJ, 116, 2196

Mayer, L. \& Moore, B. 2004, MNRAS, 354, 477

McGaugh, S. S. 1996, MNRAS, 280, 337

McGaugh, S. S. 2005, ApJ, 632, 859

McGaugh, S. S. \& de Blok, W. J. G. 1997, ApJ, 481, 689

McGaugh, S. S., Rubin, V. C., \& de Blok, W. J. G. 2001, AJ, 122, 2381

McGaugh, S. S., Schombert, J. M., Bothun, G. D., \& de Blok, W. J. G. 2000, ApJ, 533, L99

Meyer, M. J. et al. 2004, MNRAS, 350, 1195

Mo, H. J., Mao, S., \& White, S. D. M. 1998, MNRAS, 295, 319

Moore, B., Ghigna, S., Governato, F., Lake, G., Quinn, T., Stadel, J., \& Tozzi, P. 1999, ApJ, 524, L19

Mori, M. \& Burkert, A. 2000, ApJ, 538, 559

Nagashima, M. \& Yoshii, Y. 2004, ApJ, 610, 23

Norberg, P. et al. 2002, MNRAS, 332, 827

Oemler, A. 1974, ApJ, 194, 1

Read, J. I. \& Trentham, N. 2005, Royal Society of London Philosophical Transactions Series A, 363, 2693 
Sandage, A. 1975, in Stars and Stellar Systems, Vol. IX. Galaxies and the Universe, University of Chicago Press

Scannapieco, C., Tissera, P. B., White, S. D. M., \& Springel, V. 2005, MNRAS, 364, 552

Schlegel, D. J., Finkbeiner, D. P., \& Davis, M. 1998, ApJ, 500, 525

Schombert, J. M., McGaugh, S. S., \& Eder, J. A. 2001, AJ, 121, 2420

Shapiro, P. R., Iliev, I. T., \& Raga, A. C. 2004, MNRAS, 348, 753

Sheth, R. K., Mo, H. J., \& Tormen, G. 2001, MNRAS, 323, 1

Skillman, E. D. 1996, in ASP Conf. Ser. 106: The Minnesota Lectures on Extragalactic Neutral Hydrogen, ed. E. D. Skillman, 208

Spergel, D. N. et al. 2006, astro-ph/0603449

Tassis, K., Kravtsov, A. V., \& Gnedin, N. Y. 2006, ApJ, submitted

Taylor, C. L., Kobulnicky, H. A., \& Skillman, E. D. 1998, AJ, 116, 2746

Tremonti, C. A. et al. 2004, ApJ, 613, 898

van den Bosch, F. C. 2000, ApJ, 530, 177

van Zee, L. 2001, AJ, 121, 2003

Verheijen, M. A. W. 2001, ApJ, 563, 694

West, A. A. 2005, Ph.D. Thesis

Willick, J. A., Strauss, M. A., Dekel, A., \& Kolatt, T. 1997, ApJ, 486, 629

Willman, B., Dalcanton, J. J., Martinez-Delgado, D., West, A. A., Blanton, M. R., Hogg, D. W., Barentine, J. C., Brewington, H. J., Harvanek, M., Kleinman, S. J., Krzesinski, J., Long, D., Neilsen, E. H., Nitta, A., \& Snedden, S. A. 2005, ApJ, 626, L85

York, D. et al. 2000, AJ, 120, 1579

Zaritsky, D., Smith, R., Frenk, C., \& White, S. D. M. 1997, ApJ, 478, 39

Zucker, D. B. et al. 2004, ApJ, 612, L121 
Table 1. Low Mass SDSS Dwarf Galaxy Properties

\begin{tabular}{|c|c|c|c|c|c|c|c|c|c|c|c|c|c|c|c|}
\hline Name & $\begin{array}{c}\alpha(\mathrm{J} 2000) \\
(\mathrm{h}: \mathrm{m}: \mathrm{s}) \\
\end{array}$ & $\begin{array}{c}\delta(\mathrm{J} 2000) \\
\left({ }^{\circ}:^{\prime}:{ }^{\prime \prime}\right) \\
\end{array}$ & $\begin{array}{c}\text { Distance } \\
\left(h_{70}^{-1} \mathrm{Mpc}\right) \\
\end{array}$ & $\mathrm{m}_{r}$ & $M_{r}$ & $g-r$ & $\mu_{\text {eff }, r}$ & $\begin{array}{r}r_{\text {eff }, r} \\
\operatorname{kpc}\left({ }^{\prime \prime}\right)\end{array}$ & $b / a$ & $\begin{array}{c}M_{\text {stellar }} \\
(\log M \odot) \\
\end{array}$ & $\begin{array}{c}M_{\mathrm{HI}} \\
(\log M \odot) \\
\end{array}$ & $\begin{array}{c}\sigma_{M_{\mathrm{HI}}} \\
(\log M \odot) \\
\end{array}$ & $\begin{array}{r}\mathrm{W} 20 / 2 \\
\mathrm{~km} \mathrm{~s}^{-1}\end{array}$ & $\begin{array}{l}\mathrm{W} 20_{i, t} / 2 \\
\mathrm{~km} \mathrm{~s}^{-1}\end{array}$ & $\begin{array}{c}\sigma_{W 20_{i, t} / 2} \\
\mathrm{~km} \mathrm{~s}^{-1} \\
\end{array}$ \\
\hline 386141 & 000103.6 & +143448.6 & 15.3 & 16.72 & -15.12 & 0.38 & 20.3 & $0.6(7.8)$ & 0.65 & 7.82 & 7.56 & 0.08 & 28.7 & 26.6 & 4.7 \\
\hline 241112 & $00 \quad 13 \quad 38.7$ & $\begin{array}{r}+154030.5 \\
\end{array}$ & 17.9 & 17.02 & -15.26 & 0.25 & 21.5 & $1.9(21.5)$ & 0.16 & 7.79 & 8.63 & 0.08 & 71.8 & 63.8 & 1.3 \\
\hline 192963 & 003127.6 & -104033.2 & 35.1 & 17.43 & -16.16 & 0.13 & 22.5 & $1.9(11.4)$ & 0.45 & 7.96 & 8.78 & 0.04 & 85.3 & 85.0 & 6.4 \\
\hline 192971 & 003231.3 & -104121.9 & 31.4 & 17.45 & -15.90 & 0.20 & 20.7 & $1.0(6.6)$ & 0.30 & 8.00 & 8.52 & 0.05 & 52.7 & 46.0 & 4.1 \\
\hline 667743 & 003631.7 & +003347.8 & 40.2 & 17.72 & -16.14 & 0.50 & 18.8 & $0.6(3.1)$ & 0.56 & 8.43 & & & & & \\
\hline 190632 & 004751.9 & -111029.6 & 39.3 & 17.70 & -16.10 & 0.44 & 19.3 & $0.7(3.9)$ & 0.31 & 8.32 & 8.78 & 0.03 & 66.3 & 60.3 & 1.4 \\
\hline 677002 & 005756.6 & +005208.9 & 21.0 & 16.96 & -15.50 & 0.21 & 21.3 & 0.5 & 0.53 & 7.85 & 8.18 & 0.08 & 43.2 & 40.6 & 4.6 \\
\hline 47936 & 005855.5 & +134315.1 & 48.2 & 17.74 & -16.69 & 0.39 & 18.8 & $0.4(1.5)$ & 0.80 & 8.51 & 8.77 & 0.06 & 63.9 & 92.0 & 23.7 \\
\hline 677307 & $01 \quad 1420.3$ & +005500.0 & 10.7 & 16.69 & -14.31 & 0.17 & 22.1 & $0.4(8.1)$ & 0.53 & 7.32 & 8.10 & 0.08 & 54.5 & 53.8 & 2.1 \\
\hline 222989 & 011730.5 & $-09 \quad 1748.4$ & 17.6 & 15.91 & -16.19 & 0.22 & 20.8 & $1.4(16.2)$ & 0.37 & 8.10 & 8.73 & 0.06 & 70.0 & 65.6 & 1.3 \\
\hline 198542 & $\begin{array}{lll}01 & 19 & 14.3\end{array}$ & -093546.3 & 17.3 & 17.35 & -14.73 & 0.23 & 21.5 & $0.5(6.0)$ & 0.32 & 7.52 & 8.01 & 0.06 & 62.5 & 56.5 & 3.9 \\
\hline 461714 & $01 \quad 2604.7$ & +001854.9 & 17.3 & 16.33 & -15.73 & 0.20 & 21.4 & $0.9(11.2)$ & 0.39 & 7.96 & 8.66 & 0.07 & 67.8 & 63.7 & 1.3 \\
\hline 203452 & $\begin{array}{lll}01 & 27 & 25.1\end{array}$ & -084121.5 & 38.8 & 17.77 & -16.00 & 0.38 & 19.0 & $0.7(3.7)$ & 0.49 & 8.22 & & & & & \\
\hline 203478 & $01 \quad 32 \quad 22.3$ & $\begin{array}{lll}-08 & 38 & 02.4\end{array}$ & 38.6 & 17.59 & -16.18 & 0.23 & 22.4 & $1.1(5.7)$ & 0.52 & 8.17 & 8.57 & 0.03 & 53.3 & 52.3 & 1.4 \\
\hline 198720 & 014139.7 & -091303.8 & 16.5 & 16.59 & -15.35 & 0.29 & 21.5 & $0.7(8.4)$ & 0.72 & 7.86 & 7.97 & 0.07 & 39.0 & 43.7 & 2.4 \\
\hline 191112 & 015054.5 & $-1022 \quad 10.6$ & 16.4 & 17.46 & -14.50 & 0.37 & 21.4 & $0.6(7.3)$ & 0.40 & 7.66 & 8.04 & 0.07 & 54.7 & 50.1 & 2.3 \\
\hline 225456 & $\begin{array}{lll}01 & 53 & 01.4\end{array}$ & -093821.7 & 15.0 & 17.67 & -14.05 & 0.31 & 22.5 & $0.5(6.2)$ & 0.48 & 7.51 & 7.71 & 0.08 & 28.8 & 23.3 & 8.8 \\
\hline 48406 & $02 \quad 00 \quad 02.0$ & +123218.0 & 34.5 & 17.53 & -16.15 & 0.44 & 21.9 & $1.3(7.5)$ & 0.48 & 8.30 & 7.98 & 0.12 & 35.6 & 30.9 & 8.1 \\
\hline 201346 & $02 \quad 0246.9$ & $\begin{array}{llll}-08 & 27 & 27.1\end{array}$ & 21.0 & 17.07 & -15.39 & 0.41 & 22.6 & $1.2(11.4)$ & 0.39 & 7.84 & 8.22 & 0.08 & 59.1 & 54.4 & 6.3 \\
\hline 231588 & $02 \quad 0515.8$ & -093346.7 & 17.9 & 17.28 & -14.83 & 0.24 & 20.8 & $0.3(3.8)$ & 0.56 & 7.59 & 8.26 & 0.07 & 41.4 & 39.7 & 2.9 \\
\hline 227294 & $02 \quad 1756.5$ & $\begin{array}{lll}-08 & 51 & 14.2\end{array}$ & 13.5 & 16.53 & -14.98 & 0.34 & 21.5 & $0.4(6.3)$ & 0.77 & 7.77 & 7.88 & 0.07 & 45.2 & 57.2 & 2.8 \\
\hline 643417 & $\begin{array}{lll}02 & 21 & 18.9\end{array}$ & -004755.1 & 12.8 & 17.23 & -14.16 & 0.48 & 21.1 & $0.5(8.0)$ & 0.67 & 7.57 & & & & & \\
\hline 462731 & $02 \quad 52 \quad 16.8$ & +001741.3 & 14.7 & 17.21 & -14.63 & 0.23 & 21.2 & $0.3(3.6)$ & 0.99 & 7.55 & 7.81 & 0.08 & 39.0 & 301.9 & 63.0 \\
\hline 201616 & $0253 \quad 46.4$ & -072343.6 & 13.1 & 16.60 & -14.86 & 0.18 & 21.1 & $0.6(8.8)$ & 0.60 & 7.53 & 7.78 & 0.08 & 29.8 & 26.6 & 3.7 \\
\hline 467776 & $\begin{array}{lll}03 & 07 & 15.7\end{array}$ & +004352.1 & 28.7 & 17.60 & -15.74 & 0.30 & 20.7 & $0.5(3.9)$ & 0.60 & 8.09 & 8.19 & 0.09 & 46.0 & 46.6 & 5.9 \\
\hline 199465 & 033719.4 & $\begin{array}{lll}-06 & 21 & 13.2\end{array}$ & 30.5 & 17.50 & -15.81 & 0.51 & 21.7 & $1.2(8.3)$ & 0.45 & 8.14 & & 0.00 & 80.0 & 10.0 & \\
\hline 204350 & 033845.7 & -053842.1 & 31.5 & 17.61 & -15.81 & 0.36 & 19.1 & $0.5(3.0)$ & 0.54 & 8.15 & & & & & \\
\hline 161656 & $08 \quad 48 \quad 18.7$ & +011550.4 & 19.3 & 16.58 & -15.74 & 0.52 & 21.6 & $1.1(12.3)$ & 0.28 & 8.22 & 8.05 & 0.08 & 43.0 & 35.8 & 7.7 \\
\hline 123408 & $\begin{array}{lll}09 & 14 & 03.2\end{array}$ & $\begin{array}{r}+601417.3 \\
\end{array}$ & 18.7 & 16.51 & -15.75 & 0.37 & 20.4 & $0.6(6.8)$ & 0.39 & 8.09 & 7.81 & 0.08 & 29.8 & 23.2 & 2.3 \\
\hline 121139 & $09 \quad 1532.0$ & +594948.7 & 16.3 & 16.67 & -15.27 & 0.75 & 22.1 & $0.7(8.3)$ & 0.89 & 8.29 & & & & & \\
\hline 132909 & 091858.6 & $\begin{array}{r}+581407.8 \\
\end{array}$ & 14.0 & 16.86 & -14.73 & 0.27 & 22.0 & $0.6(8.3)$ & 0.40 & 7.61 & 8.26 & 0.09 & 63.3 & 59.2 & 1.4 \\
\hline 136373 & $09 \quad 2753.7$ & +602420.3 & 15.8 & 16.66 & -15.20 & 0.35 & 21.3 & $0.5(6.5)$ & 0.49 & 7.84 & 7.53 & 0.09 & 26.9 & 21.3 & 3.3 \\
\hline 276603 & 095435.7 & +042307.9 & 19.7 & 17.20 & -15.21 & 0.20 & 21.7 & $0.7(6.8)$ & 0.57 & 7.72 & 8.25 & 0.07 & 50.8 & 51.2 & 6.6 \\
\hline 232890 & 095455.0 & +563628.4 & 19.3 & 16.93 & -15.32 & 0.27 & 21.2 & $0.5(5.3)$ & 0.36 & 7.78 & 8.20 & 0.07 & 61.7 & 56.5 & 1.9 \\
\hline 169071 & 100425.1 & +023331.4 & 13.5 & 17.22 & -14.28 & 0.26 & 22.2 & $0.6(8.7)$ & 0.48 & 7.43 & 7.05 & 0.20 & 12.9 & 5.5 & 3.4 \\
\hline 278622 & $\begin{array}{lll}10 & 07 & 04.5\end{array}$ & +050024.6 & 19.2 & 16.63 & -15.63 & 0.35 & 20.4 & $1.2(12.9)$ & 0.56 & 8.07 & 8.13 & 0.08 & 32.8 & 29.5 & 6.0 \\
\hline 262647 & 101702.3 & +033845.6 & 12.8 & 16.22 & -15.15 & 0.35 & 19.9 & $0.6(9.5)$ & 0.55 & 7.86 & 7.56 & 0.14 & 31.1 & 27.2 & 7.4 \\
\hline 133217 & 102024.3 & $\begin{array}{r}+632623.7\end{array}$ & 20.8 & 16.96 & -15.43 & 0.43 & 21.3 & $1.1(10.8)$ & 0.17 & 8.04 & 8.25 & 0.06 & 63.3 & 55.3 & 2.2 \\
\hline 565755 & $\begin{array}{lll}10 & 27 & 01.8\end{array}$ & +561614.4 & 11.2 & 15.42 & -15.60 & 0.28 & 20.1 & $0.4(7.8)$ & 0.56 & 7.96 & 8.25 & 0.11 & 47.2 & 46.5 & 2.7 \\
\hline 114699 & 103126.8 & +641525.8 & 20.9 & 16.94 & -15.48 & 0.47 & 19.7 & $0.4(4.2)$ & 0.62 & 8.15 & & & & & \\
\hline 274726 & 103201.3 & +042045.9 & 13.8 & 17.75 & -13.85 & 0.32 & 22.2 & $0.3(4.8)$ & 0.56 & 7.36 & 7.77 & 0.09 & 42.8 & 41.4 & 6.3 \\
\hline 317892 & 103951.5 & $\begin{array}{r}+564359.2 \\
\end{array}$ & 14.4 & 16.45 & -15.14 & 0.20 & 21.5 & $1.1(15.6)$ & 0.35 & 7.68 & 8.80 & 0.09 & 70.0 & 65.0 & 1.5 \\
\hline 119086 & 105034.8 & +660242.4 & 14.2 & 16.46 & -15.11 & 0.39 & 21.7 & $0.7(10.4)$ & 0.35 & 7.91 & 7.83 & 0.10 & 37.4 & 30.8 & 2.9 \\
\hline 237224 & $\begin{array}{ll}1105 & 53.7\end{array}$ & +602229.2 & 16.9 & 16.29 & -15.64 & 0.12 & 21.9 & $0.8(9.7)$ & 0.42 & 7.75 & 8.84 & 0.08 & 71.5 & 68.7 & 3.0 \\
\hline 280272 & $\begin{array}{lll}11 & 08 & 22.0\end{array}$ & $\begin{array}{r}+055325.7\end{array}$ & 25.7 & 17.68 & -15.25 & 0.19 & 21.2 & $0.9(6.9)$ & 0.19 & 7.73 & 8.48 & 0.06 & 62.9 & 54.9 & 3.8 \\
\hline 246454 & 111405.2 & +021154.8 & 15.6 & 16.53 & -15.32 & 0.31 & 21.8 & $1.0(12.8)$ & 0.28 & 7.83 & 8.14 & 0.08 & 66.3 & 59.7 & 7.4 \\
\hline 319836 & $\begin{array}{ll}11 & 22 \\
35.7\end{array}$ & $\begin{array}{r}+585840.9 \\
\end{array}$ & 16.0 & 16.37 & -15.46 & 0.23 & 19.7 & $0.5(6.5)$ & 0.45 & 7.89 & 8.19 & 0.09 & 45.4 & 41.0 & 1.8 \\
\hline 327197 & $\begin{array}{lll}11 & 25 & 05.4\end{array}$ & +040715.6 & 17.6 & 17.04 & -15.12 & 0.21 & 21.1 & $0.6(7.1)$ & 0.31 & 7.68 & 8.58 & 0.06 & 74.2 & 68.4 & 1.7 \\
\hline 327205 & $\begin{array}{lll}11 & 26 & 08.3\end{array}$ & $\begin{array}{r}040344.5 \\
\end{array}$ & 16.9 & 16.45 & -15.58 & 0.18 & 21.1 & $0.6(7.0)$ & 0.62 & 7.88 & 8.08 & 0.06 & 39.0 & 39.0 & 3.1 \\
\hline 323534 & 113014.4 & +595626.9 & 13.1 & 16.45 & -14.95 & 0.34 & 21.7 & $1.0(15.4)$ & 0.58 & 7.63 & 8.27 & 0.10 & 55.4 & 57.2 & 1.6 \\
\hline
\end{tabular}


Table 1-Continued

\begin{tabular}{|c|c|c|c|c|c|c|c|c|c|c|c|c|c|c|c|}
\hline Name & $\begin{array}{c}\alpha(\mathrm{J} 2000) \\
(\mathrm{h}: \mathrm{m}: \mathrm{s}) \\
\end{array}$ & $\begin{array}{c}\delta(\mathrm{J} 2000) \\
\left({ }^{\circ}:^{\prime}:{ }^{\prime \prime}\right) \\
\end{array}$ & $\begin{array}{c}\text { Distance } \\
\left(h_{70}^{-1} \mathrm{Mpc}\right) \\
\end{array}$ & $\mathrm{m}_{r}$ & $M_{r}$ & $g-r$ & $\mu_{\text {eff }, r}$ & $\begin{array}{c}r_{\mathrm{eff}, r} \\
\operatorname{kpc}\left({ }^{\prime \prime}\right)\end{array}$ & $b / a$ & $\begin{array}{c}M_{\text {stellar }} \\
(\log M \odot) \\
\end{array}$ & $\begin{array}{c}M_{\mathrm{HI}} \\
(\log M \odot)\end{array}$ & $\begin{array}{c}\sigma_{M_{\mathrm{HI}}} \\
(\log M \odot)\end{array}$ & $\begin{array}{r}\mathrm{W} 20 / 2 \\
\mathrm{~km} \mathrm{~s}^{-1}\end{array}$ & $\begin{array}{l}\mathrm{W} 20_{i, t} / 2 \\
\mathrm{~km} \mathrm{~s}^{-1}\end{array}$ & $\begin{array}{c}\sigma_{W 20_{i, t} / 2} \\
\mathrm{~km} \mathrm{~s}^{-1}\end{array}$ \\
\hline 330228 & 113518.4 & $+0457 \quad 17.4$ & 15.9 & 16.99 & -14.87 & 0.36 & 21.7 & $0.4(5.4)$ & 0.59 & 7.75 & 7.95 & 0.08 & 35.4 & 33.4 & 6.0 \\
\hline 72997 & 115328.6 & -031347.2 & 15.9 & 16.17 & -15.66 & 0.32 & 20.6 & $0.4(5.8)$ & 0.62 & 8.00 & 8.13 & 0.08 & 49.8 & 52.0 & 2.6 \\
\hline 325105 & 115349.0 & +605209.8 & 15.8 & 16.13 & -15.74 & 0.55 & 19.0 & $0.6(8.0)$ & 0.61 & 8.30 & & & & & \\
\hline 64798 & 115503.6 & $\begin{array}{lll}-03 & 30 & 12.2\end{array}$ & 15.5 & 17.53 & -14.26 & 0.33 & 21.1 & $0.3(3.6)$ & 0.71 & 7.47 & 7.33 & 0.08 & 28.5 & 28.7 & 4.1 \\
\hline 67068 & $1157 \quad 12.4$ & $-0241 \quad 11.3$ & 15.3 & 16.61 & -15.16 & 0.16 & 21.5 & $0.7(9.3)$ & 0.32 & 7.61 & 8.01 & 0.08 & 25.4 & 18.1 & 2.4 \\
\hline 328956 & $\begin{array}{lll}11 & 59 & 00.8\end{array}$ & +044010.7 & 17.5 & 16.37 & -15.69 & 0.32 & 19.6 & $0.3(4.0)$ & 0.67 & 8.03 & 7.87 & 0.07 & 35.2 & 35.9 & 4.3 \\
\hline 535294 & 115943.3 & +533639.1 & 14.4 & 16.57 & -15.05 & 0.37 & 21.8 & $0.7(9.4)$ & 0.56 & 7.83 & 7.52 & 0.10 & 22.7 & 17.5 & 4.2 \\
\hline 238933 & 120243.3 & +622952.4 & 13.3 & 15.82 & -15.62 & 0.26 & 21.8 & $1.0(14.8)$ & 0.26 & 7.96 & 8.34 & 0.10 & 61.2 & 54.1 & 1.9 \\
\hline 166775 & 120820.0 & +023019.9 & 20.7 & 16.66 & -15.76 & 0.31 & 19.8 & $0.3(3.0)$ & 1.00 & 8.05 & 8.32 & 0.06 & 62.7 & & \\
\hline 158190 & $12 \quad 1847.1$ & +012253.6 & 17.5 & 17.69 & -14.37 & 0.24 & 22.1 & $0.6(7.5)$ & 0.34 & 7.39 & 7.80 & 0.06 & 27.4 & 20.2 & 3.6 \\
\hline 296747 & 121949.9 & +640524.7 & 17.5 & 16.89 & -15.17 & 0.55 & 18.8 & $0.6(6.5)$ & 0.53 & 8.06 & & & & & \\
\hline 172859 & $12 \quad 2054.7$ & +032411.7 & 22.5 & 17.10 & -15.49 & 0.22 & 21.4 & $0.6(5.2)$ & 0.45 & 7.86 & 8.18 & 0.05 & 56.5 & 53.3 & 2.2 \\
\hline 149339 & 122330.0 & +020029.1 & 19.2 & 16.72 & -15.54 & 0.31 & 21.3 & $1.4(14.8)$ & 0.15 & 7.96 & 8.25 & 0.08 & 62.5 & 54.5 & 10.3 \\
\hline 258802 & $12 \quad 2435.4$ & +644234.9 & 18.1 & 16.77 & -15.35 & 0.51 & 20.6 & $0.7(8.4)$ & 0.30 & 8.13 & & & & & \\
\hline 148003 & 123246.4 & +013408.9 & 16.7 & 16.98 & -14.97 & 0.21 & 22.2 & $0.7(8.2)$ & 0.49 & 7.55 & 8.14 & 0.07 & 30.8 & 25.7 & 1.4 \\
\hline 329114 & 123258.8 & +043445.0 & 13.9 & 15.92 & -15.62 & 0.41 & 22.8 & $1.3(19.2)$ & 0.45 & 7.83 & 8.30 & 0.08 & 51.0 & 47.3 & 1.9 \\
\hline 66304 & 123643.7 & $\begin{array}{lll}-03 & 01 & 14.4\end{array}$ & 25.3 & 16.85 & -16.04 & 0.20 & 20.9 & $0.6(4.9)$ & 0.44 & 8.09 & 8.23 & 0.08 & 56.7 & 53.2 & 18.7 \\
\hline 67359 & 123747.0 & -023159.4 & 24.3 & 16.71 & -16.08 & 0.35 & 19.8 & $0.6(4.7)$ & 0.47 & 8.23 & & & & & \\
\hline 73362 & $\begin{array}{lll}12 & 41 & 28.9\end{array}$ & $\begin{array}{lll}-03 & 15 & 13.4\end{array}$ & 18.8 & 16.85 & -15.38 & 0.45 & 20.8 & $1.0(11.2)$ & 0.15 & 7.98 & 8.04 & 0.06 & 62.8 & 54.8 & 2.5 \\
\hline 6716 & 125253.2 & -004921.7 & 15.8 & 16.72 & -15.12 & 0.37 & 21.1 & $0.7(8.7)$ & 0.32 & 7.86 & 7.53 & 0.08 & 31.2 & 24.1 & 4.1 \\
\hline 32636 & 125405.1 & $\begin{array}{lll}-00 & 06 & 04.2\end{array}$ & 10.9 & 15.99 & -15.02 & 0.54 & 21.6 & $0.6(11.3)$ & 0.93 & 7.98 & 7.10 & 0.13 & 28.2 & 53.5 & 9.0 \\
\hline 45831 & 130240.8 & +010426.8 & 10.4 & 16.92 & -13.97 & 0.28 & 20.6 & $0.3(5.9)$ & 0.70 & 7.30 & 7.20 & 0.15 & 35.0 & 37.4 & 16.5 \\
\hline 148433 & 132955.8 & +013238.4 & 12.0 & 15.99 & -15.25 & 0.28 & 21.9 & $0.5(9.1)$ & 0.72 & 7.80 & 7.94 & 0.10 & 33.7 & 36.2 & 3.4 \\
\hline 346768 & $1344 \quad 17.3$ & +611409.0 & 21.4 & 16.87 & -15.61 & 0.27 & 21.7 & $1.2(11.7)$ & 0.31 & 7.88 & 7.90 & 0.06 & 38.8 & 31.8 & 3.8 \\
\hline 370242 & 141911.5 & -021515.6 & 25.8 & 17.60 & -15.35 & 0.10 & 20.5 & $0.6(5.1)$ & 0.34 & 7.63 & 8.19 & 0.04 & 43.3 & 36.9 & 3.7 \\
\hline 381017 & 142230.7 & $-01 \quad 1344.4$ & 18.5 & 16.78 & -15.45 & 0.27 & 22.3 & $1.0(11.2)$ & 0.31 & 7.94 & 8.00 & 0.06 & 44.9 & 38.2 & 2.5 \\
\hline 371747 & 142704.8 & -014346.8 & 18.8 & 17.67 & -14.62 & 0.26 & 21.1 & $0.4(3.9)$ & 0.46 & 7.54 & 7.82 & 0.06 & 35.1 & 30.0 & 3.6 \\
\hline 178719 & 143822.6 & +043647.8 & 17.8 & 17.35 & -14.81 & 0.34 & 21.1 & $0.5(5.6)$ & 0.35 & 7.73 & 7.86 & 0.08 & 72.0 & 67.1 & 7.4 \\
\hline 301586 & 144133.7 & +032948.0 & 17.7 & 16.50 & -15.61 & 0.52 & 19.3 & $1.0(11.8)$ & 0.49 & 8.20 & 7.40 & 0.08 & 34.6 & 30.0 & 14.7 \\
\hline 307723 & $1446 \quad 20.2$ & +044358.4 & 16.4 & 17.56 & -14.40 & 0.18 & 22.0 & $0.5(5.8)$ & 0.35 & 7.33 & 7.80 & 0.06 & 34.1 & 27.4 & 4.6 \\
\hline 377686 & 145620.3 & -024541.8 & 19.4 & 17.96 & -14.53 & 0.15 & 22.2 & $0.4(4.6)$ & 0.71 & 7.44 & 7.68 & 0.06 & 27.7 & 27.5 & 3.9 \\
\hline 373377 & 150933.6 & $\begin{array}{lll}-01 & 01 & 17.5\end{array}$ & 19.1 & 17.09 & -15.28 & 0.40 & 12.9 & $1.7(18.0)$ & 0.53 & 7.97 & & & & & \\
\hline 284191 & 151306.3 & +565808.8 & 11.4 & 15.77 & -15.33 & 0.46 & 22.4 & $0.9(15.9)$ & 0.43 & 8.11 & 8.41 & 0.10 & 53.1 & 49.1 & 1.6 \\
\hline 379759 & 153239.2 & -013606.2 & 26.4 & 17.80 & -15.48 & 0.29 & 21.5 & $0.9(6.8)$ & 0.78 & 7.93 & 8.89 & 0.04 & 107.7 & 156.1 & 12.6 \\
\hline 377995 & 153829.6 & -022346.2 & 31.1 & 17.82 & -15.84 & 0.11 & 21.3 & $0.8(5.1)$ & 0.80 & 7.89 & 8.73 & 0.04 & 48.6 & 67.0 & 2.2 \\
\hline 340807 & 160938.8 & $\begin{array}{r}+451642.8 \\
\end{array}$ & 19.8 & 16.55 & -15.76 & 0.29 & 22.4 & 1.8 (18.3) & 0.49 & 7.99 & 8.77 & 0.05 & 67.5 & 67.1 & 2.2 \\
\hline 208160 & 204156.9 & $-0517 \quad 56.5$ & 34.0 & 17.89 & -15.70 & 0.46 & 19.8 & $0.3(2.1)$ & 0.84 & 8.14 & 8.12 & 0.05 & 51.2 & 78.8 & 8.9 \\
\hline 398936 & $21 \quad 1537.4$ & -002431.3 & 32.0 & 17.37 & -16.10 & 0.63 & 22.4 & $0.9(5.5)$ & 0.93 & 8.39 & 8.07 & 0.07 & 21.1 & 34.4 & 7.0 \\
\hline 217927 & 212006.0 & +115506.5 & 11.7 & 15.72 & -15.63 & 0.38 & 19.5 & $0.3(5.6)$ & 0.55 & 8.11 & 8.31 & 0.07 & 79.2 & 83.7 & 1.3 \\
\hline 209838 & 212202.3 & +095310.6 & 28.9 & 17.19 & -16.04 & 0.36 & 19.5 & $0.4(2.6)$ & 0.79 & 8.22 & 7.82 & 0.13 & 43.4 & 56.4 & 34.2 \\
\hline 189157 & 214839.9 & -065011.4 & 28.7 & 17.45 & -15.71 & 0.29 & 21.0 & $0.6(4.3)$ & 0.55 & 8.03 & & & & & \\
\hline 181782 & 222100.1 & $-0938 \quad 30.2$ & 27.7 & 17.40 & -15.72 & 0.03 & 22.0 & $1.1(8.1)$ & 0.22 & 7.80 & 8.71 & 0.04 & 68.6 & 61.1 & 2.4 \\
\hline 421204 & 223036.8 & -000637.0 & 15.3 & 17.06 & -14.83 & 0.11 & 11.0 & $0.4(5.1)$ & 0.85 & 7.37 & 7.84 & 0.08 & 39.1 & 57.4 & 7.4 \\
\hline 186498 & 223925.1 & $-0845 \quad 57.7$ & 38.7 & 17.85 & -16.04 & 0.30 & 21.6 & $1.1(5.9)$ & 0.62 & 8.19 & 8.30 & 0.05 & 53.9 & 57.6 & 7.6 \\
\hline 392224 & 225924.7 & -102916.2 & 31.6 & 17.56 & -15.84 & 0.34 & 22.4 & $1.2(7.6)$ & 0.70 & 8.14 & 8.34 & 0.07 & 30.6 & 31.1 & 5.0 \\
\hline 410317 & 230120.5 & -005533.3 & 28.7 & 17.20 & -16.00 & 0.31 & 21.5 & $0.9(6.1)$ & 0.34 & 8.16 & 8.35 & 0.09 & 64.4 & 58.8 & 21.2 \\
\hline 213776 & $\begin{array}{lll}23 & 05 & 11.2\end{array}$ & +140347.4 & 14.3 & 16.52 & -15.57 & 0.31 & 21.1 & $0.3(4.7)$ & 0.72 & 7.95 & 7.90 & 0.07 & 52.1 & 62.0 & 4.9 \\
\hline 183623 & 230556.3 & -100257.0 & 21.3 & 17.07 & -15.47 & 0.11 & 21.5 & $0.5(5.2)$ & 0.64 & 7.67 & 8.33 & 0.07 & 40.1 & 41.0 & 5.0 \\
\hline 215437 & 230615.1 & +143927.4 & 14.2 & 16.88 & -15.19 & 0.27 & 22.5 & $0.5(6.8)$ & 0.87 & 7.77 & 8.48 & 0.07 & 54.7 & 92.0 & 1.8 \\
\hline 633306 & $\begin{array}{lll}23 & 28 & 12.3\end{array}$ & -010346.2 & 24.5 & 17.43 & -15.38 & 0.13 & 21.8 & $0.8(6.5)$ & 0.53 & 7.67 & 8.35 & 0.06 & 41.9 & 39.3 & 4.6 \\
\hline
\end{tabular}


Table 1 - Continued

\begin{tabular}{|c|c|c|c|c|c|c|c|c|c|c|c|c|c|c|c|}
\hline Name & $\begin{array}{r}\alpha(\mathrm{J} 2000) \\
(\mathrm{h}: \mathrm{m}: \mathrm{s}) \\
\end{array}$ & $\begin{array}{c}\delta(\mathrm{J} 2000) \\
\left({ }^{\circ}:^{\prime}:{ }^{\prime \prime}\right) \\
\end{array}$ & $\begin{array}{c}\text { Distance } \\
\left(h_{70}^{-1} \mathrm{Mpc}\right) \\
\end{array}$ & $\mathrm{m}_{r}$ & $M_{r}$ & $g-r$ & $\mu_{\mathrm{eff}, r}$ & $\begin{array}{r}r_{\text {eff }, r} \\
\operatorname{kpc}\left({ }^{\prime \prime}\right) \\
\end{array}$ & $b / a$ & $\begin{array}{c}M_{\text {stellar }} \\
(\log M \odot) \\
\end{array}$ & $\begin{array}{c}M_{\mathrm{HI}} \\
(\log M \odot) \\
\end{array}$ & $\begin{array}{c}\sigma_{M_{\mathrm{HI}}} \\
(\log M \odot) \\
\end{array}$ & $\begin{array}{r}\mathrm{W} 20 / 2 \\
\mathrm{~km} \mathrm{~s}^{-1} \\
\end{array}$ & $\begin{array}{l}\mathrm{W} 20_{i, t} / 2 \\
\mathrm{~km} \mathrm{~s}^{-1}\end{array}$ & $\begin{array}{c}\sigma_{W 20_{i, t} / 2} \\
\mathrm{~km} \mathrm{~s}^{-1} \\
\end{array}$ \\
\hline 421787 & 233102.1 & -000755.0 & 24.1 & 17.16 & -15.63 & 0.36 & 21.7 & $1.0(8.5)$ & 0.29 & 8.02 & 8.60 & 0.05 & 61.6 & 54.9 & 2.3 \\
\hline
\end{tabular}

Note. - Galaxy names are based on position in the Blanton et al. (2005) low luminosity SDSS catalog. Absolute magnitudes and $g-r$ colors have been corrected for Galactic extinction based on Schlegel et al. (1998). Distances are estimated based on a model of the local velocity field (Willick et al. 1997) and assume $\mathrm{H}_{0}=70 h_{70} \mathrm{Mpc}_{\mathrm{km} \mathrm{s}} \mathrm{s}^{-1}$. The effective surface brightness ( $\mu_{\text {eff }}, r$ ), effective half-light radius $\left(r_{\text {eff,r }}\right)$ and axis ratio $(b / a)$ are measured from the SDSS $r$-band images. Stellar masses are calculated based on Bell et al. (2003) and a Kroupa et al. (1993) initial mass function; HI masses are based on our Arecibo and GBT observations. The HI velocity half-width, W20/2, is the observed uncorrected $20 \%$ peak $\mathrm{HI}$ half-width. The quantity W20 $\mathrm{i}_{i, t} / 2$ is the $\mathrm{HI}$ half-with recovered from Monte Carlo simulations. Galaxies which were not detected in the radio do not have HI properties listed in this table. 
Table 2. Tully-Fisher Relation Fit Parameters

\begin{tabular}{lrrrr}
\hline \hline \multicolumn{1}{c}{ Galaxy Sample } & \multicolumn{1}{c}{$a$} & \multicolumn{1}{c}{$b$} & \multicolumn{1}{c}{$\sigma$} \\
\hline Stellar mass (current sample) & $-0.14 \pm 0.02$ & $0.43 \pm 0.06$ & 0.12 \\
Baryonic mass (current sample) & $-0.32 \pm 0.05$ & $0.53 \pm 0.08$ & 0.13 \\
$M_{I} \quad$ (full sample) & $0.37 \pm 0.01$ & $-0.09 \pm 0.01$ & 0.11 \\
Baryonic mass (full sample) & $-0.19 \pm 0.02$ & $0.27 \pm 0.01$ & 0.10 \\
\hline
\end{tabular}

Note. - Tully-Fisher linear regression fit parameters for Equation 3. The zero-point $a$ is in units $\log _{10}\left(\mathrm{~W} 20_{i, t} / 2 /\left(50 \mathrm{~km} \mathrm{~s}^{-1}\right)\right)$. The slope $b$ is in units $\log _{10}\left(\operatorname{mass} / 10^{8} h_{70}^{-2} M_{\odot}\right)$ per $\log _{10}\left(\mathrm{~W} 20_{i, t} / 2 /\left(50 \mathrm{~km} \mathrm{~s}^{-1}\right)\right)$. In the $I$-band Tully-Fisher relationship we replace $\log _{10}\left(\operatorname{mass} / 10^{8} h_{70}^{-2} M_{\odot}\right)$ with $M_{I}-5 \log _{10} h_{70}+20$. The quantity $\sigma$ represents the logarithmic (base 10) scatter around each fit. The first two rows ('current sample') are fits to the galaxy data presented in this paper; the last two rows ('full sample') are fits to this sample plus data from the literature as desribed in $\S 3.2$. 

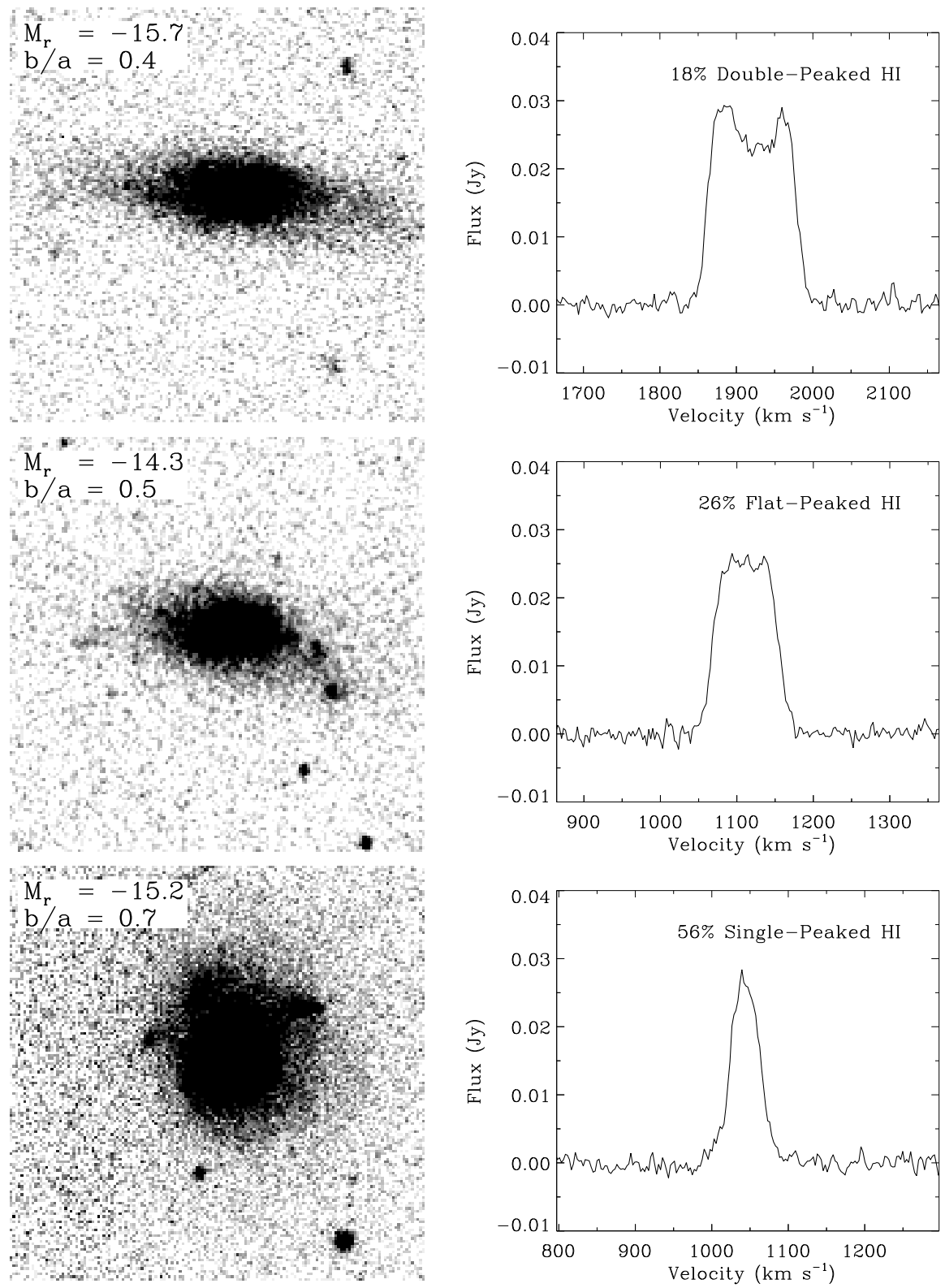

Fig. 1.- SDSS $r$-band images and HI line-widths for three representative dwarf galaxies. The images are $1^{\prime}$ on a side. We list the absolute $r$-band magnitude and optically measured axis ratio $(b / a)$ for each galaxy. Of the dwarf galaxy sample presented here, $18 \%$ have doublepeaked HI profiles similar to the top panel, $26 \%$ have flat-topped HI profiles, and $56 \%$ are single peaked profiles. If we consider only edge-on galaxies $(b / a<0.4)$, the percentage of double-peaked profiles increases to $30 \%$. The distribution of HI profile shapes suggests that the majority of dwarf galaxies in our sample have regular rotation fields. 

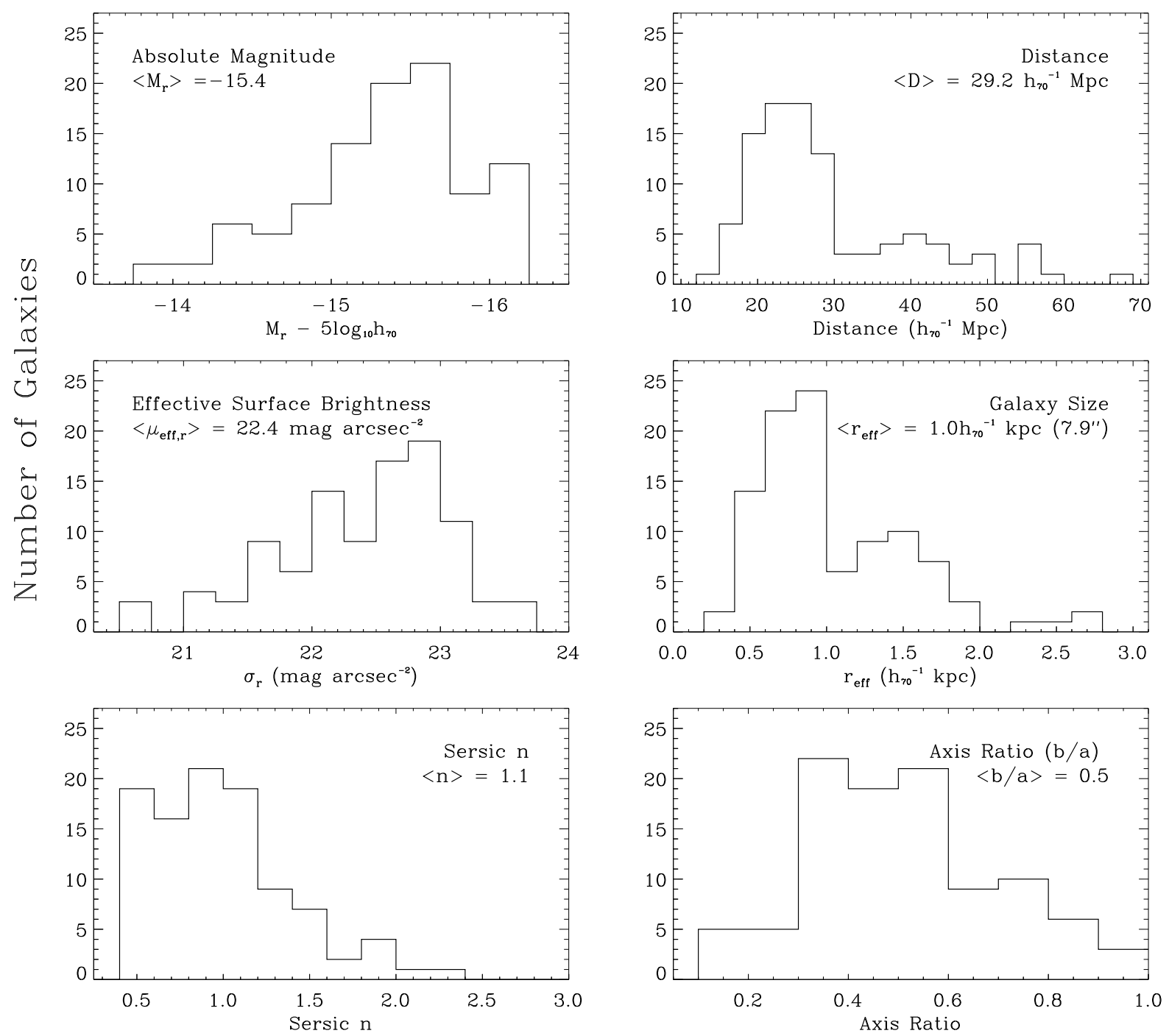

Fig. 2.- The distribution ( top left to bottom right) of absolute magnitude, distance, effective surface brightness, effective half-light radius, Sérsic index and axis ratio for our dwarf galaxy sample. All photometric parameters are measured from the SDSS $r$-band images. Distances are estimated based on a model of the local velocity field (Willick et al. 1997). In each panel, we list the average value of each quantity for the 101 dwarf galaxies in this sample. 


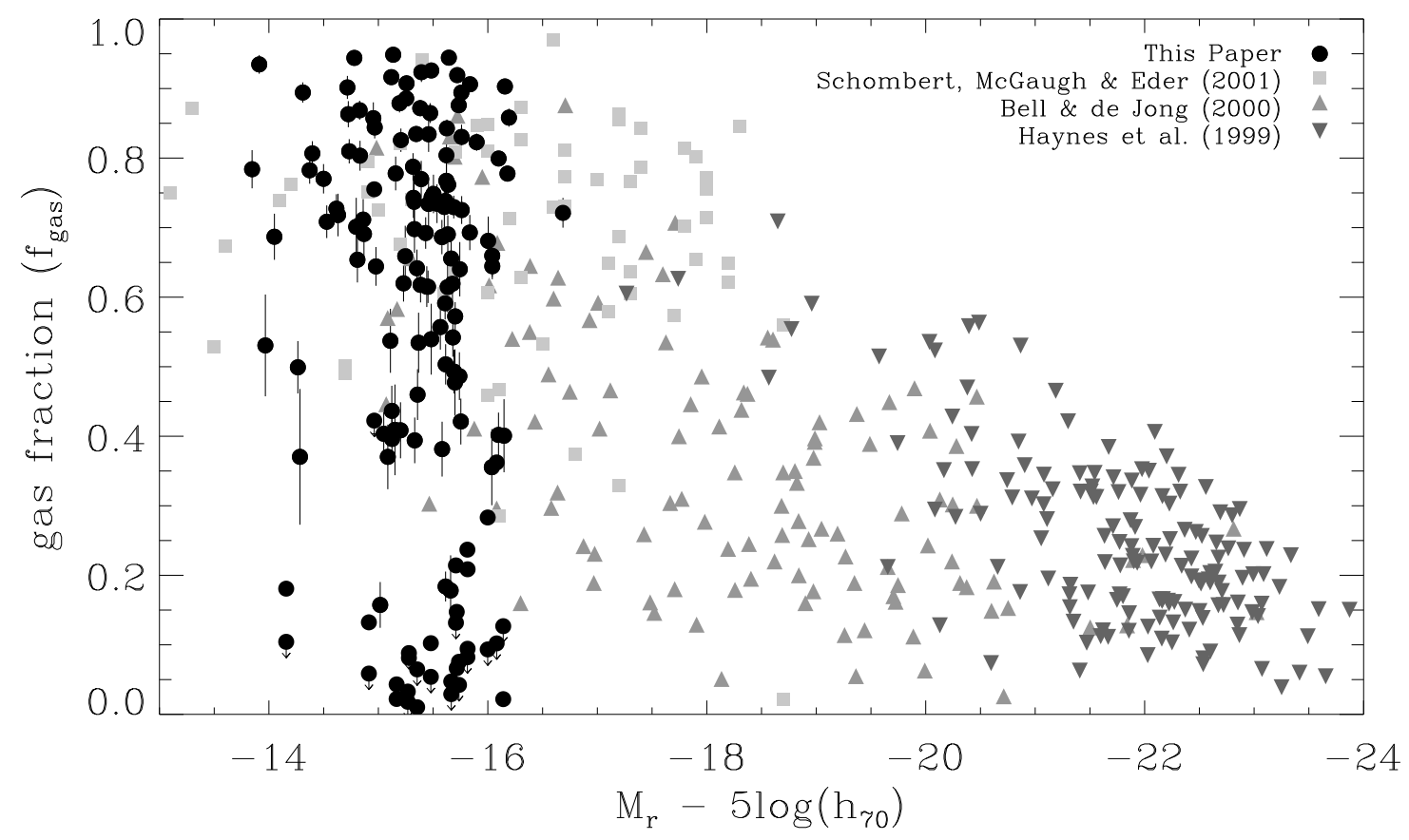

Fig. 3.- The gas fraction, $f_{\text {gas }}=M_{\text {gas }} /\left(M_{\text {gas }}+M_{\text {stellar }}\right)$, plotted as a function of absolute $r$ band magnitude. The majority of dwarf galaxies in this paper (black circles) are dominated by the gas mass, rather than stellar mass, with gas fractions as high as $95 \%$. Galaxies which were not detected in HI are plotted as upper limits. For comparison, we plot data from Schombert et al. (2001, squares), Bell \& de Jong (2000, upward triangles) and Haynes et al. (1999, downward triangles). We infer from this plot that, compared to more luminous galaxies, dwarf galaxies are far less efficient at turning gas into stars over their lifetimes. 

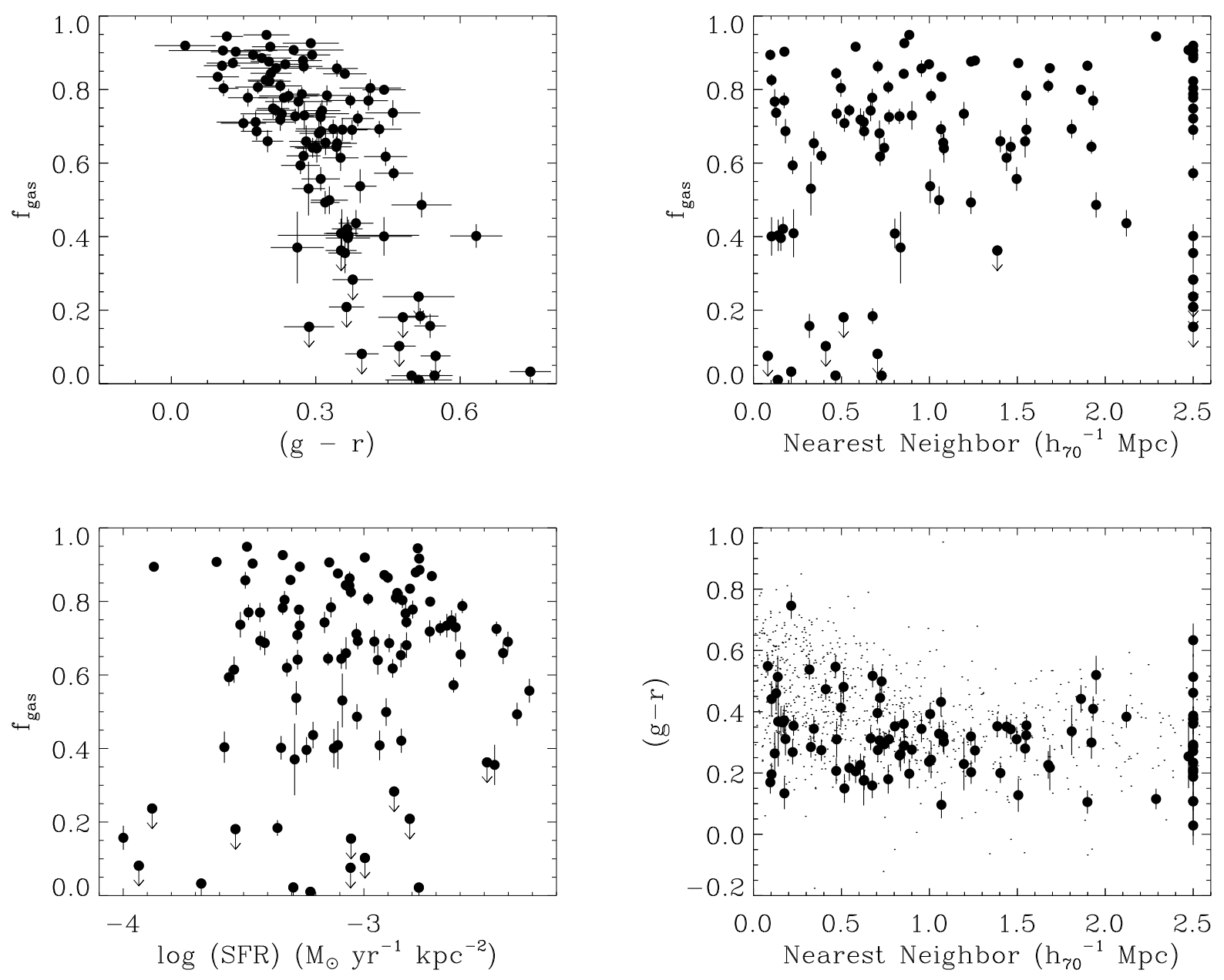

Fig. 4.- Top Left: The gas fraction, $f_{\text {gas }}$, plotted as a function of $g-r$ color. Assuming the $g-r$ color traces recent star-formation activity, the fraction of star-formation occurring in the recent past is higher for the gas-rich galaxies in our sample. Bottom Left: In contrast, the current star formation rate per unit area, as measured by the SDSS $u$-band flux, is not correlated with gas fraction. Top Right: The gas fraction plotted against the projected distance to the nearest luminous neighbor. Galaxies with gas fractions $f_{\text {gas }}<0.4$ are preferentially found within $0.5 \mathrm{Mpc}$ of a luminous neighbor galaxy. The gas fractions of galaxies with $f_{\text {gas }}>0.4$ are uncorrelated with nearest neighbor distance. Bottom Right: We also compare the $g-r$ color and nearest neighbor distance distribution of our sample to that of the parent SDSS dwarf galaxy catalog (small black symbols). 

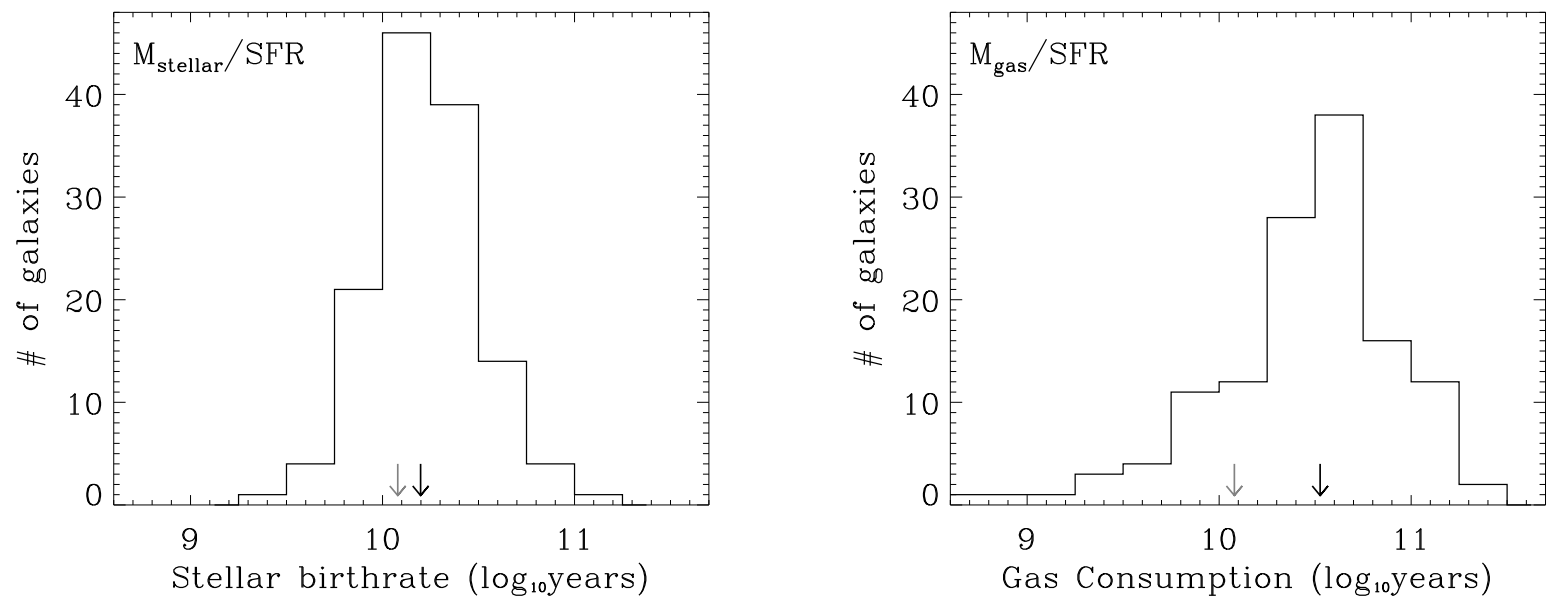

Fig. 5.- Left: The stellar birthrate timescale defined as $M_{\text {stellar }} / S F R_{\text {tot }}$. The median birthrate timescale for this dwarf sample is $15 \mathrm{Gyr}$ (black arrow). Assuming a single galaxy formation time of $\sim 12$ Gyr (grey arrow), roughly half the galaxies in our sample had lower star formation rates and half have had higher rates in the past than presently observed. This suggests that star formation in dwarfs is stochastic and that the current star formation rate is not necessarily representative of the time-average rate. Right: The large gas consumption timescale $\left(M_{\text {gas }} / S F R_{\text {tot }}\right)$ suggests that the majority of galaxies will be able to make stars at the current rates for another Hubble time. 

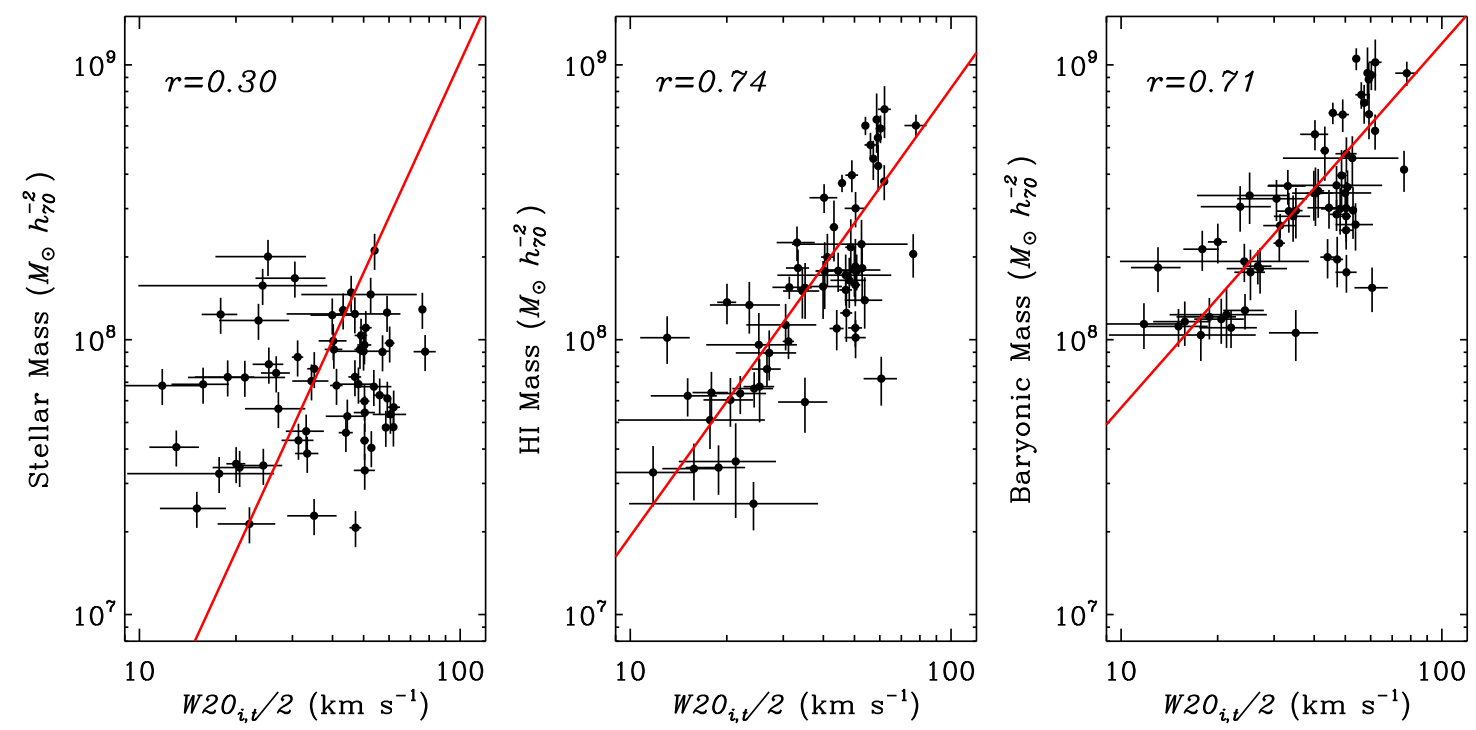

Fig. 6. - The stellar mass, HI mass, and total baryonic mass plotted as a function of rotation velocity as measured by the inclination- and turbulence-corrected HI half line-widths $\left(\mathrm{W} 20_{i, t} / 2\right)$. The solid line is the (inverse) regression fit described in $\S 3.2$, whose parameters are quoted in Table 2. Each panel lists the correlation coefficient $r$ between the two quantities; the correlation coefficient is zero for two unrelated quantities and unity for two perfectly linearly related quantities. The scatter in the stellar mass relationship (left) is much larger compared to the HI (middle) and baryonic Tully-Fisher relationship (right). 

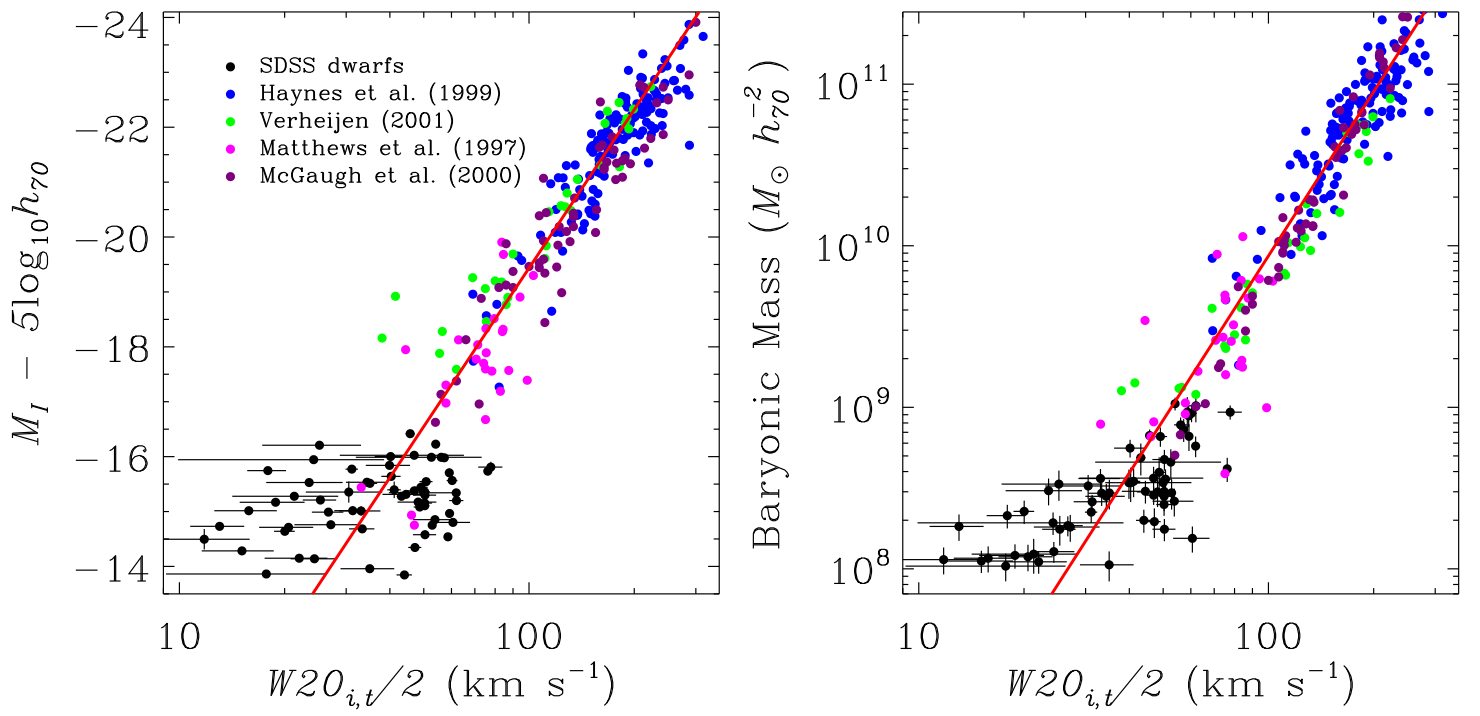

Fig. 7.- The optical and baryonic Tully-Fisher relationships: absolute $I$-band luminosity (left) and the total baryonic mass (right) plotted as a function of maximum rotational velocity $\left(\mathrm{W} 20_{i, t} / 2\right)$. We compare our dwarf galaxy sample to literature data for which HI and optical measurements are available: Haynes et al. (1999), Verheijen (2001), Matthews et al. (1998), and McGaugh et al. (2000). The line in each panel is the fit we describe in the text. The slope in the right-hand panel $b=0.27 \pm 0.01$ is consistent with the CDM prediction $b \sim 0.29$ (Bullock et al. 2001). 

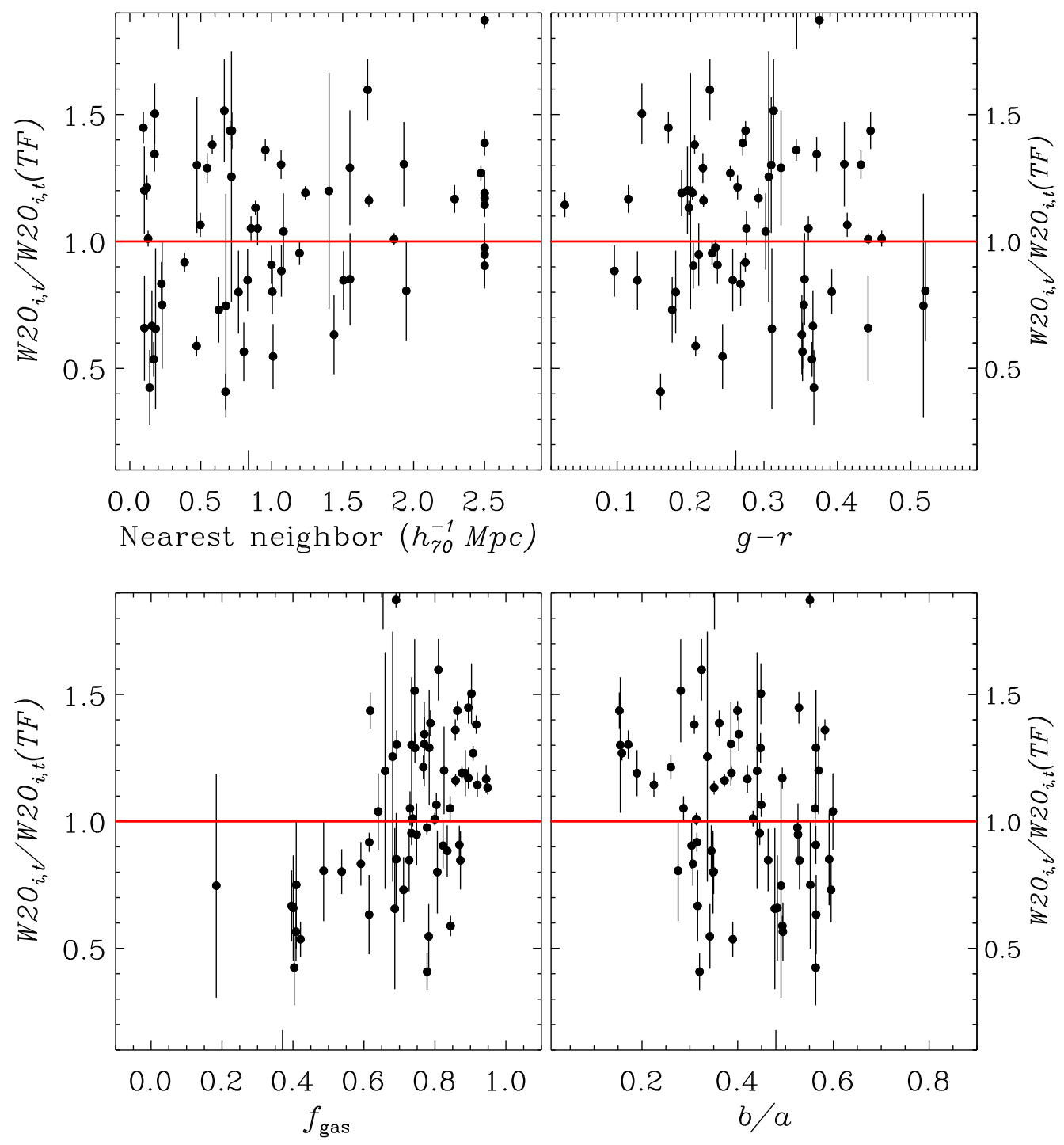

Fig. 8.- Residuals from the baryonic Tully-Fisher relationship, defined as the ratio $W 20_{i, t} / W 20_{i, t}(T F)$ of the actual line width to the expected line width from the Tully-Fisher relationship. We show this ratio versus nearest neighbor distance, $g-r$ color, gas fraction $f_{\text {gas }}$ and optical axis ratio $b / a$. 